Math. Model. Nat. Phenom.

Vol. 6, No. 5, 2011, pp. 98-129

DOI: $10.1051 / \mathrm{mmnp} / 20116506$

\title{
Microscopic Modelling of Active Bacterial Suspensions
}

\author{
A. Decoene ${ }^{1}$, S. Martin and B. Maury \\ Université Paris-Sud 11, Département de Mathématiques, Bâtiment 425, 91405 Orsay cedex (France)
}

\begin{abstract}
We present two-dimensional simulations of chemotactic self-propelled bacteria swimming in a viscous fluid. Self-propulsion is modelled by a couple of forces of same intensity and opposite direction applied on the rigid bacterial body and on an associated region in the fluid representing the flagellar bundle. The method for solving the fluid flow and the motion of the bacteria is based on a variational formulation written on the whole domain, strongly coupling the fluid and the rigid particle problems: rigid motion is enforced by penalizing the strain rate tensor on the rigid domain, while incompressibility is treated by duality. This model allows to achieve an accurate description of fluid motion and hydrodynamic interactions in moderate to concentrated active suspensions. A mesoscopic model is also used, in which the size of the bacteria is supposed to be much smaller than the elements of fluid: the perturbation of the fluid due to propulsion and motion of the swimmers is neglected, and the fluid is only subjected to the buoyant forcing induced by the presence of the bacteria, which are denser than the fluid. Although this model does not accurately take into account hydrodynamic interactions, it is able to reproduce complex collective dynamics observed in concentrated bacterial suspensions, such as bioconvection. From a mathematical point of view, both models lead to a minimization problem which is solved with a standard Finite Element Method. In order to ensure robustness, a projection algorithm is used to deal with contacts between particles. We also reproduce chemotactic behaviour driven by oxygen: an advection-diffusion equation on the oxygen concentration is solved in the fluid domain, with a source term accounting for oxygen consumption by the bacteria. The orientations of the individual bacteria are subjected to random changes, with a frequency that depends on the surrounding oxygen concentration, in order to favor the direction of the concentration gradient.
\end{abstract}

Key words: Stokes flow, fluid-particle flows, self-propulsion, finite element method, penalty method, chemotaxis.

\footnotetext{
${ }^{1}$ Corresponding author. E-mail: astrid.decoene@ math.u-psud.fr
} 
AMS subject classification: 70E55, 74F10, 76Z05, 92B05

\section{Individual and collective dynamics of self-propelled chemo- tactic bacteria}

\subsection{Individual swimming and chemotactic behaviour}

We focus on flagellated bacteria that are able to swim in a viscous fluid by means of self-propulsion, as Escherichia coli (E. coli) and Bacillus subtilis (B. subtilis). These are very common bacteria that can be found in digestive tract of animals, or in soil and water. They are so small that, on their scale, the fluid is essentially viscous and inertial effects can be neglected when describing their locomotion. In fact, these microorganisms live in the low Reynolds regime $\left(R e \sim 10^{-2}\right)$ (see [2], [19] and references therein).

E. coli and B. subtilis are among the most studied and well-understood bacteria, and they represent important model organisms in many fields of research, particularly molecular biology, genetics, biochemistry. Individual cells of these bacteria are rod-shaped (length about $2 \mu \mathrm{m}$ for E. coli, about $4 \mu \mathrm{m}$ for $B$. subtilis, diameter of the order of $1 \mu \mathrm{m}$ for both), and their surface is peritrichously flagellated. The helical flagella, responsible for propulsion, are significantly longer than the cell's body: approximately $20 \mathrm{~nm}$ in diameter, and $10-15 \mu \mathrm{m}$ long. For E. coli, the flagellar filaments are four or five. They are distributed randomly over the cell's body, attached to motors that are fixed within the cell membrane $[19,33]$. When the motor turns counter-clockwise, the filaments wrap into a bundle that pushes the cell along at speeds of 25 to $35 \mu \mathrm{m} \mathrm{s}^{-1}$. When one or more of the motors reverse, the swimming direction of the cell can change.

As described by Berg [1,2], these bacteria alternate two basic behavioral modes: a more or less linear motion called a run, and a highly erratic motion called tumble, the purpose of which is to reorient the cell. During a run the bacterium moves in the most recently chosen direction. Then it tumbles: it randomly chooses a new direction and starts moving straightforward again. That is how these bacteria perform chemotaxis, which means swimming up a chemoattractant gradient $[1,25]$. Run times are typically much longer than the time spent tumbling, and they are closely related to the surrounding environment. In fact, when bacteria move in a favorable direction (i.e. in the direction of a nutrient concentration gradient) the run times are increased; on the contrary, they are decreased when the direction is not favorable. These bacteria are too small to detect instantaneously spatial differences in the concentration of an attractant at their own length scale. Therefore they measure if they are going in the right direction by doing several successive evaluations of the concentration in time. The effect of alternating these two modes of behavior, and in particular, of increasing the run duration when moving in a favorable direction, is that a bacterium executes a three-dimensional random walk with drift in a favorable direction when observed on a sufficiently long time scale.

Note that many flagellated bacteria can also move on the surface of certain solid media. Their 
rapid and organized propagation on these surfaces is known as swarming, and strongly depends on cell-to-cell contact and reversal of direction (see for instance [6] and [17]). We will not consider this behaviour in this work since we focus on hydrodynamic interactions, but there are interesting common aspects in both swimming and swarming motility.

\subsection{Collective dynamics in bacterial suspensions}

The individual swimming mechanism and the chemotactic behaviour of self-propelled bacteria give rise to interesting and complex collective dynamics and large-scale motions in bacterial suspensions. This has been highlighted through many experiments, as for example those in $[5,8,19$, $31,36]$, and has created important concern during the last decade. In particular, there has been a great interest in understanding if and how small scale swimming could generate large scale correlation. It is believed that the essential ingredients in the formation of large-scale patterns are hydrodynamic interactions between individual swimmers and the environment, including boundary effects and cell-to-cell interaction, as well as gravity and tumbling.

The easiest observable patterns in a bacterial suspension, especially in simulations (see Section 4), are recirculations and whirls at a scale which is much larger than the individual bacterium. This can already be observed at moderate concentration. But when the bacterial cells are very concentrated, or nearly close-packed, they form codirectionally swimming structures that move chaotically, giving the appearance of turbulence $[5,36]$. The velocity of the flow can also be significantly enhanced by collective dynamics. In [31], experimental studies have revealed collective flow speeds that exceed the speed of the individual bacterium by an order of magnitude. This phenomenon can be essential to the survival of the organisms, since they allow an enhanced molecular transport into the bacterial suspension. In the case of oxygen, for example, which is consumed by the bacteria, diffusion may not be sufficient in absence of transport to supply the suspension.

Besides, the presence of microscopic swimmers can strongly modify the rheological properties of a fluid. This has been well established in the case of passive inclusions. But experimental $[27,30]$ and numerical studies $[21,25]$ have shown that suspensions of active inclusions, i.e. inclusions which are submitted to some internal force, may have completely different properties. In [21], the apparent viscosity of a mixture of a Newtonian fluid and interacting particles has been evaluated through numerical simulations. This has shown that the viscosity of the fluid can be significantly enhanced or decreased, depending on the interaction force between the particles. In [30], experiments show that self-propelled bacteria can reduce the viscosity of the suspension up to five times compared to passive bacteria. The rheology properties of suspensions of self-propelled bacteria have recently been extensively studied by Berlyand and coauthors in $[3,12]$ : they have considered different models for the individual bacteria, and analysed the resulting suspensions analytically in the dilute regime and numerically in the moderate concentration regime. These studies show that the decrease in the effective viscosity of the suspension is due not only to self-propulsion, but also to hydrodynamical interactions, which can lead to an alignment of bacteria with the flow. In fact, alignment is essential to the decrease of the effective viscosity (see for instance [25]).

Bioconvection is another important issue of collective dynamics in suspensions of swimming microorganisms. In a shallow suspension, due to chemotaxis, the cells swim upward toward the 
oxygen-rich air/fluid interface. Since bacteria are approximately 10 percent denser than water, accumulation at the interface results in an unstable gradient of mean fluid density, creating convective dynamics. In a horizontal meniscus, these unstable stratifications evolve through a Rayleigh Taylor instability in falling plumes, dragging the swimmers from the surface into the bulk fluid [25]. We have focused on an experiment by Dombrowski and coauthors [8], in which bioconvective dynamics in a suspension of $B$. subtilis in a sessile drop are displayed. The experiment shows in particular the influence of the geometry of the drop on these dynamics. Bacteria swim up to the free surface and, under the influence of gravity, slide down the slanted meniscus. This generates a persistent hydrodynamic vortex, which confines the bacteria near the contact line and promotes the advection of oxygen into the suspension. Besides, instabilities in the accumulation layer appear in the flatter part of the drop, consistent with the instability of bioconvection in fluids with a horizontal meniscus [25].

In [34], a macroscopic model for chemotactic self-propelled bacteria was introduced, that reproduces numerically the bioconvective dynamics observed in the experiment described previously. The model consists of three coupled equations describing the dynamics of the oxygen concentration, cell concentration and fluid velocity. The first one is an advection-diffusion equation with a sink term accounting for consumption of oxygen by the bacteria, and the second one consists of the Navier-Stokes equations with buoyant forcing, accounting for the density variations caused by bacteria. The equation describing the dynamics of the cell concentration is as follows :

$$
\frac{\partial \rho}{\partial t}+\mathbf{u} \cdot \nabla \rho=D_{n} \Delta \rho-\nabla \cdot(f(c) \rho \nabla c)
$$

where $\rho$ is the cell concentration, $\mathbf{u}$ is the fluid velocity, $D_{n}$ is the bacterial diffusion constant and $f(c)$ is the chemosensitivity function that modulates motility, so that $f(c) \nabla c$ represents the chemotactic velocity of the cells.

The present approach is based on a microscopic description of those phenomena. By microscopic we mean that bacteria are considered individually, and not represented as a global density. A wide hierarchy of such microscopic models can actually be considered (see e.g. [13, 26, 28]), depending on the way the hydrodynamic interactions are handled. We also refer to [37] for a microscopic model describing swarming dynamics. To allow for suitable balancing between accuracy in the description of hydrodynamic effects and computational cost constraints, we propose here two models, at both ends of this hierarchy. The first one (see Section 2.1.) is fully microscopic: the bacteria are embodied in the computational domain, and direct simulation of Stokes flow is performed at their very scale. In order to capture alignment phenomena, and to give a realistic description of the shape of the bacteria, we have modelled bacteria as elongated elliptic rigid particles. As we shall see this fully microscopic model, which is computationally expensive, is necessary to reproduce low turbulence phenomena which are observed in dense suspensions. The second approach (see Section 2.2.), which we shall refer to as mesoscopic, lies inbetween the microscopic and the macroscopic models. The swimmers are supposed to be so small that the perturbation of the fluid due to their propulsion and motion is neglected, and the fluid is only subjected to buoyant forcing due to the presence of bacteria. This model, based on a cruder description of entities and therefore less expensive, allows to qualitatively reproduce bioconvective dynamics, as 
those displayed in the experiment by Dombrovski and coauthors (see Section 4). This model is mesoscopic in the sense that fluid computations shall be performed at a scale which is considered much larger than the size of the perturbation induced by the motion of a single particle. Yet it keeps some microscopic features: in particular, each bacterium is described individually, as the model integrates the run-and-tumble cycles for individual particles. Besides, it could be enriched to account more accurately for hydrodynamic interactions (e.g. by integrating Stokesian dynamics tools more sophisticated than the simple Faxen law).

\subsection{Outline of the paper}

In the next section, we describe the different models used for our simulations: first, the fully microscopic model (Section 2.1), then the mesoscopic model (Section 2.2), the stochastic model used to represent the run and tumble cycles (Section 2.3), and finally the model for oxygen dynamics (Section 2.4). In Section 3 we present the numerical procedure used to solve the fluid-particle interaction problem, including the way we handle contacts beween particles, and the discrete version of the stochastic model for tumbling. Finally, Section 4 is devoted to the presentation of the simulation results, obtained using both the fully microscopic and the mesoscopic models, coupled (or not) to the oxygen dynamics in the fluid and the run and tumble cycles.

\section{Mathematical models}

\subsection{Fully microscopic model}

We consider a connected bounded and regular domain $\Omega \subset \mathbb{R}^{2}$ and we denote by $\left(B_{i}\right)_{i=1, \ldots, N}$ the rigid particles, strongly included in $\Omega$ (see right in Fig.1). $B$ denotes the whole rigid domain: $B=\cup_{i} B_{i}$. The domain $\Omega \backslash \bar{B}$ is filled with Newtonian fluid governed by the Stokes equations. Here, $\mu$ is the viscosity of the fluid and $\mathbf{f}_{f}$ the external forces exerted on it. Since we consider a Newtonian fluid, the stress tensor $\underline{\underline{\sigma}}$ writes

$$
\underline{\underline{\sigma}}=2 \mu \mathbb{D}(\mathbf{u})-p \mathbb{I}, \quad \text { where } \quad \mathbb{D}(\mathbf{u})=\frac{\nabla \mathbf{u}+(\nabla \mathbf{u})^{T}}{2}
$$

and $p$ is the pressure. For the sake of simplicity we will consider homogeneous Dirichlet conditions on $\partial \Omega$. On the other hand, viscosity imposes a no-slip condition on the boundary $\partial B$ of the rigid domain.

In [7], following [3], we modelled bacteria as spheres, with an associated point force representing the action of flagella. Here, in order to better take into account the elongated shape of the swimmers, we model the bacterial bodies as ellipses. Self-propulsion is modelled by a couple of forces of same intensity and opposite direction applied on the rigid bacterial body and on a region in the fluid next to the body representing the flagellar bundle. In fact, self-propulsion is characterized by a balance of forces, unlike the case of propulsion due to external forces, which are 

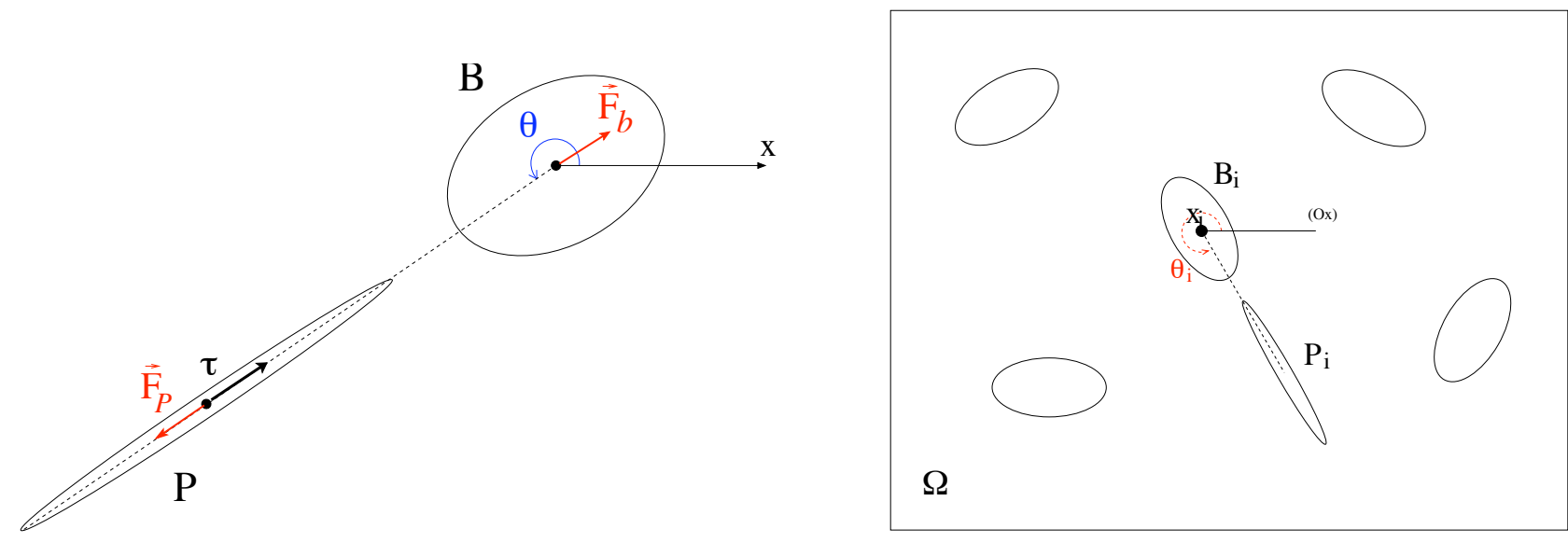

Figure 1: Left: single bacterium, right: whole domain with inclusions

unbalanced. It is important to note that, while the bacterial bodies are handled as rigid elongated particles moving in the fluid, the flagella are not materialized in our model. We only take into account the force they exert on the particles and on the fluid. In that framework, we have chosen to model the force exerted by the flagellar bundle on the fluid as a volume force density $\mathbf{f}_{p}$ supported in a very elongated ellipse $P$, placed at a constant distance from the bacterial body. Spreading out the Dirac force in that way makes sense from a modelling point of view: the actual force is exerted by a bunch of flagella which move in a complex way, and that results in an effective force acting on the fluid in a zone downstream the bacterium with non-zero volume. The propulsion force is directed outward from the center of $B$, parallel to the major semiaxes of $B$ and $P$, and has some orientation angle $\theta$, measured from the $x$-axis, as shown in Fig. 1 .

The total force exerted on the fluid by the flagella associated to the $i$ th particle has a constant magnitude $f_{p}$ and will be denoted by $\mathbf{F}_{p}^{i}$ :

$$
\mathbf{F}_{p}^{i}=-f_{p} \boldsymbol{\tau}_{i}=\int_{P_{i}} \mathbf{f}_{P}^{i} \mathrm{~d} \mathbf{x}, \quad \text { with } \quad \mathbf{f}_{p}^{i}=-\frac{f_{p}}{\operatorname{meas}\left(P_{i}\right)} \boldsymbol{\tau}_{i},
$$

where $\boldsymbol{\tau}_{i}$ is given by the orientation angle $\theta_{i}$ and meas $\left(P_{i}\right)$ denotes the measure of the ellipse $P_{i}$. The total force exerted by the flagella on their associated particle $B_{i}$ will be denoted by $\mathbf{F}_{b}^{i}$ and is equal to $-\mathbf{F}_{p}^{i}$, so that we can write:

$$
\mathbf{F}_{b}^{i}=f_{p} \boldsymbol{\tau}_{i}=\int_{B_{i}} \mathbf{f}_{b}^{i} \mathrm{~d} \mathbf{x}, \quad \text { with } \quad \mathbf{f}_{b}^{i}=\frac{f_{p}}{\operatorname{meas}\left(B_{i}\right)} \boldsymbol{\tau}_{i} .
$$

At the initial time the particles are distributed randomly over the fluid (without overlapping). The position of the center of the $i$ th particle is denoted by $\mathbf{x}_{i}$, and by $\mathbf{v}_{i}$ and $\omega_{i}$ its translational and angular velocities.

We have to find the velocity $\mathbf{u}=\left(u_{1}, u_{2}\right)$ and the pressure field $p$ defined in $\Omega \backslash \bar{B}$, as well as the velocities of the particles $\mathbf{V}:=\left(\mathbf{v}_{i}\right)_{i=1, \ldots, N} \in \mathbb{R}^{2 N}$ and $\boldsymbol{\omega}:=\left(\omega_{i}\right)_{i=1, \ldots, N} \in \mathbb{R}^{N}$ such that: 


$$
\left\{\begin{aligned}
-\mu \Delta \mathbf{u}+\nabla p & =\mathbf{f}_{f} & & \text { in } \Omega \backslash \bar{B} \\
\nabla \cdot \mathbf{u} & =0 & & \text { in } \Omega \backslash \bar{B} \\
\mathbf{u} & =\mathbf{0} & & \text { on } \partial \Omega \\
\mathbf{u} & =\mathbf{v}_{i}+\omega_{i} \times\left(\mathbf{x}-\mathbf{x}_{i}\right) & & \text { on } \partial B_{i}, \quad \forall i \in\{1, \ldots, N\} .
\end{aligned}\right.
$$

The forces considered on the fluid are the forces exerted by the flagella, so that

$$
\mathbf{f}_{f}=\sum_{i=1, \ldots, N} \mathbf{f}_{p}^{i} \chi_{p}^{i},
$$

where $\chi_{p}^{i}$ is the characteristic function associated to the flagellar bundle $P_{i}$. Finally, Newton's second law of motion, written here in the non-inertial regime, couples the equations:

$$
\left\{\begin{array}{r}
\int_{B_{i}} \rho \mathbf{g}+\int_{B_{i}} \mathbf{f}_{b}^{i}-\int_{\partial B_{i}} \underline{\underline{\sigma}} \cdot \mathbf{n}=\mathbf{0} \quad \forall i \\
\int_{\partial B_{i}}\left(\mathbf{x}-\mathbf{x}_{i}\right) \times \underline{\underline{\sigma}} \cdot \mathbf{n}=\mathbf{0} \quad \forall i
\end{array}\right.
$$

where $\rho$ denotes the buoyant density of bacteria, which is positive as bacteria are denser than the fluid, and $\mathbf{g}=(0,-g)$ is gravity.

The motion of each bacterium $B_{i}$ is then set by its instantaneous velocity $\mathbf{u}(\mathbf{x}, t)=\mathbf{v}_{i}(t)+$ $\omega_{i}(t) \times\left(\mathbf{x}-\mathbf{x}_{i}(t)\right)$ defined on $\partial B_{i}$. On one hand, the center $\mathbf{x}_{i}$ of the particle $B_{i}$ follows the differential equation

$$
\dot{\mathbf{x}}_{i}(t)=\mathbf{v}_{i}(t) .
$$

On the other hand the relative position of its corresponding flagellar bundel $P_{i}$ rotates with angular velocity $\omega_{i}$, i.e.

$$
\dot{\theta}_{i}(t)=\omega_{i}(t)
$$

This model describes the dynamics of self-propelled particles in a Stokes flow, and thus it describes the hydrodynamic interactions between particles (i.e. their interaction through the fluid). One can wonder what happens when they get very close to each other, especially in the case of dense suspensions. Assuming, as we have in this model, that particle surfaces are smooth and that Stokes model is valid at any scale, it is known that contacts are not supposed to happen (see $[9,15])$. In fact, two passive or active particles can get infinitely close to each other, but they will never collide. Therefore, solving the equations of this model exactly would lead to interactions between particles without contact. However, this is no longer true when we solve the time and space approximation of these equations, and therefore we have to deal with possible collisions in the numerical simulations. We send the reader to section 3.4 for a description of the method used to avoid contact between particles numerically. 


\subsection{A mesoscopic model}

We consider here that the swimmers are much smaller than the scale at which fluid flow is described. The solid phase is represented as:

$$
\rho=\sum_{i=1, \ldots, N} m \delta_{x_{i}},
$$

where $m$ is the buoyancy mass of one bacterium and $\delta_{x_{i}}$ is the delta function related to the position of $i$-th cell. The equilibrium of forces applied on the body of each bacterium $i$ writes:

$$
\mathbf{F}_{b}^{i}+\beta\left(\mathbf{u}\left(\mathbf{x}_{i}\right)-\mathbf{v}_{i}\right)+m \mathbf{g}=\mathbf{0},
$$

where $\beta$ denotes the coefficient relating the hydrodynamic force exerted on the body to its relative velocity, $\mathbf{u}\left(\mathbf{x}_{i}\right)$ is the velocity of the fluid at the position of the bacterium $i$, and $\mathbf{v}_{i}$ is the velocity of the swimmer. We recall that following Faxen's law (see for instance [14, 18]), for a sphere in an unbounded Stokes flow, $\beta=6 \pi \mu R_{b}$, where $R_{b}$ is the radius of the sphere.

Since the size of bacteria is very small compared to the fluid elements, we neglect the effect of the propulsion force and the swimmer's motion on the fluid: the latter is only subjected to buoyant forcing induced by the presence of the bacteria. Thus, the model consists in solving the following problem in the fluid domain:

$$
\left\{\begin{aligned}
-\mu \Delta \mathbf{u}+\nabla p & =\sum_{i=1, \ldots, N} m \mathbf{g} \delta_{x_{i}} & & \text { in } \Omega, \\
\nabla \cdot \mathbf{u} & =0 & & \text { in } \Omega, \\
\mathbf{u} & =\mathbf{0} & & \text { on } \partial \Omega .
\end{aligned}\right.
$$

Following (2.5), the velocity of each particle $i$ is then given by :

$$
\mathbf{v}_{i}=\mathbf{u}\left(\mathbf{x}_{i}\right)+\frac{1}{\beta}\left(\mathbf{F}_{b}^{i}+m \mathbf{g}\right) .
$$

The position of each particle $B_{i}$ will follow the differential equation (2.3).

Let us make it clear that this model is somewhat ambiguous, for its level of accuracy strongly depends on the way discretization parameters are chosen (i.e. typical mesh step size), compared to the typical interparticle distance. We shall consider here coarse discretizations: the mesh diameter shall not be small compared to this distance, so that the fluid actually sees the solid phase as a macroscopic density. The same model could be used in a different manner (closer to the full microscopic model) by using a fine discretization : in this case, local perturbations induced by single bacteria can be reproduced by the numerical model, only perturbations due to the swimming process (which are an order of magnitude smaller) are neglected. To make a step further toward full microscopic description, yet keeping a punctual description of entities, one could account for these perturbations by adding a doublet force term $\mathbf{F}_{b}^{i}\left(\delta_{x}-\delta_{y}\right)$ for each bacterium, $y$ denoting some average position of the flagella.

Yet, as we shall see, despite the crude assumptions upon which it is based, the model just described allows to reproduce the basic features of the collective behaviour observed in bioconvection experiments. 


\subsection{Modelling tumbling and chemotaxis}

The bacteria we consider perform chemotaxis by alternating running and tumbling (see Section 1.1), and biasing the duration of the runs. The run durations are exponentially distributed with a rate that depends on the environment. In the case of $E$. coli, for example, the tumbling rate is of average $\lambda \sim 1 \mathrm{~s}^{-1}$ in isotropic medium (see [24]). Therefore, we model tumbling through a Poisson process with variable intensity. The time spent tumbling is neglected, and the run time $T_{\text {run }}$ of each bacterium, i.e. the time elapsed between two tumbles, follows an exponential law of parameter $\lambda$, which represents the tumbling frequency. This frequency varies depending on the surrounding environment of the bacterium, that is depending on if it is going in a favorable direction or not.

Let $c$ be the concentration of the chemical attractant considered. We use a simple model in which the tumbling frequency is determined by the sign of the Lagrangian derivative $\mathrm{D} c / \mathrm{D} t$ of the attractant concentration along the trajectory of the bacterium :

$$
\lambda(t)=\lambda_{0}\left(1-\varepsilon \phi\left(\frac{\mathrm{D} c}{\mathrm{D} t}\right)\right)
$$

were $\lambda_{0}$ is the average tumbling rate and $\phi$ is the sign function. Note that this model doesn't take into account the memory of bacteria relatively to the concentration of attractant along their trajectory. Finer models have been used to deal with memory (see for instance [24] and [29]).

Although the swimming directions before and after a tumble are correlated, with a mean angle between both directions of $\sim 60^{\circ}[1,24]$, we neglect this fact and, in our model, bacteria choose a new orientation angle $\theta$ which is uniformly distributed over $(0,2 \pi)$ after each run. However, persistence in the re-orientation seems to have non-neglectable effects, as analysed by Locsei and Pedley in [24].

\subsection{Oxygen dynamics}

In this work we consider oxygen as the only chemoattractant in the bacteria's environment. The dynamics of the oxygen concentration $c$ are described by the following equation:

$$
\partial_{t} c+\mathbf{u} \cdot \nabla c-D_{c} \Delta c=-g(c, B)
$$

where $D_{c}$ is the oxygen diffusion coefficient, $\mathbf{u}$ the fluid velocity and $-g(c, B)$ is the sink term accounting for the oxygen consumption by the bacteria. Following [34], this term is defined through

$$
g(c, B)=\kappa f(c) \chi_{B},
$$

where $\kappa$ is the volumic consumption rate (in oxygen molecules per second per volume of bacteria), $\chi_{B}$ is the characteristic function for the rigid ("bacterial") domain, and $c \rightarrow f(c)$ modulates this rate, equal to 1 when $c$ is large, but vanishing as $c$ is smaller than a critical value. This modulation allows to verify the minimum principle on the oxygen concentration.

The motion of the bacteria is also related to the oxygen concentration, since bacteria which do not consume enough oxygen loose their capacity of self-propulsing. Therefore, the intensity 
of the self-propulsion force $f_{p}$ is modulated by a function called the motility function $r(c)$ (see again [34]), which is equal to one at large $c$ and vanishes rapidly for small $c$. In our numerical simulations we have taken

$$
r(c)=\frac{\arctan \left(k c / c^{*}\right)}{\arctan k},
$$

where $c^{*}$ is the maximal value of the concentration in the domain and $k=10$.

\subsection{Coupling the hydrodynamic model with tumbling and oxygen dynamics}

The hydrodynamic model is coupled to chemotaxis through the individual reorientation (tumbling) process just described. Concretely, each bacterium has a translation and rotation movement determined by the hydrodynamic model (see equations (2.3)-(2.4)). But it additionally tumbles at a frequency $\lambda(t)$ which is given by equation (2.8) and depends on the sign of the oxygen concentration gradient on the bacterium trajectory. When it tumbles, a bacterium changes its position following equation (2.3) and randomly chooses a new orientation instead of rotating following equation (2.4).

\section{Numerical procedure}

\subsection{Variational formulation for the fully microscopic model}

We want to treat the fluid-particle interaction problem (2.1)-(2.2) in a strongly coupled way. In addition, we want to avoid working on the time dependent fluid domain, what would necessitate remeshing. Therefore, following $[16,20]$, we write a variational formulation of the continuous problem involving functions which are defined on the whole domain $\Omega$.

Let us first introduce the following constrained functional spaces:

$$
\begin{aligned}
& K_{\nabla}=\left\{\mathbf{u} \in H_{0}^{1}(\Omega), \nabla \cdot \mathbf{u}=0\right\} \\
& K_{B}=\left\{\mathbf{u} \in H_{0}^{1}(\Omega), \forall i \exists\left(\mathbf{v}_{i}, \omega_{i}\right) \in \mathbb{R}^{2} \times \mathbb{R} ; \mathbf{u}(\mathbf{x})=\mathbf{v}_{i}+\omega_{i} \times\left(\mathbf{x}-\mathbf{x}_{i}\right) \text { a.e. in } B_{i}\right\} .
\end{aligned}
$$

$K_{\nabla}$ is the space of divergence free functions defined on $\Omega$, and $K_{B}$ is the space of functions which do not deform $B$. We will rather use the equivalent characterisation $\mathbb{D}(\mathbf{u})=0$ for rigid motion (see for instance [32]), so that:

$$
K_{B}=\left\{\mathbf{u} \in H_{0}^{1}(\Omega), \mathbb{D}(\mathbf{u})=0 \text { a.e. in } B\right\} .
$$

The solution to the initial problem, defined on $\Omega \backslash \bar{B}$, can be extended on the whole domain $\Omega$ by a function in $K_{B}: \mathbf{u}(\mathbf{x}, \cdot)=\mathbf{v}_{i}+\omega_{i} \times\left(\mathbf{x}-\mathbf{x}_{i}\right)$ in $B_{i} \forall i$, and we still denote this extension by $\mathbf{u}$.

Let $(\mathbf{u}, p)$ be the extended solution of the problem. On one hand, we multiply the momentum equation (2.1) by a test function $\widetilde{\mathbf{u}}$ in $K_{B}$ and integrate it by parts over $\Omega \backslash \bar{B}$. Using the fact that 
$\nabla \cdot \mathbf{u}=0$ and $\mathbb{D}(\mathbf{u})=0$ in $B$, the integrals can be extended over $\Omega$. Finally, using the boundary conditions of the problem and writing the incompressibility equation in its usual variational form, we obtain the following variational formulation:

$$
\left\{\begin{aligned}
2 \mu \int_{\Omega} \mathbb{D}(\mathbf{u}): \mathbb{D}(\widetilde{\mathbf{u}})-\int_{\Omega} p \nabla \cdot \widetilde{\mathbf{u}} & =\int_{\Omega} \mathbf{f} \cdot \widetilde{\mathbf{u}}, & & \forall \widetilde{\mathbf{u}} \in K_{B} \\
\int_{\Omega} q \nabla \cdot \mathbf{u} & =0, & & \forall q \in L_{0}^{2}(\Omega),
\end{aligned}\right.
$$

where

$$
\mathbf{f}=\sum_{i=1}^{N}\left(\mathbf{f}_{b}^{i} \chi_{b}^{i}+\mathbf{f}_{p}^{i} \chi_{p}^{i}\right)
$$

and $L_{0}^{2}(\Omega)$ stands for the set of $L^{2}$ functions over $\Omega$ with zero mean value. Functions $\chi_{p}^{i}$ and $\chi_{b}^{i}$ are, respectively, the characteristic function associated to the flagellar bundle $P_{i}$ and to the particle $B_{i}$.

\subsection{Penalty method to enforce the rigid motion}

As mentioned before, we do not want to mesh the fluid domain: the complexity of the mesh due to the presence of inclusions would no allow to use standard fast solvers, and the computational cost of remeshing could become prohibitive. Therefore, we choose the fictitious domain approach, which has been used for fluid-particle interactions by several authors. One possibility is to use an iterative algorithm on an auxiliary field, composed by Lagrange multipliers, which warrants the rigid motion constraint of the particles (see for instance $[10,11]$ ). An alternative to this are penalty methods: the rigid motion constraint is obtained by relaxing a term in the variational formulation, what amounts to replacing rigid zones by highly viscous ones (see $[35,16,20]$ ). In this work we choose a penalty method already used in [20] for the simulation of passive particles in a fluid.

Formulation (3.1) involves a solution and test functions defined in the constrained space of rigid motions on $B$. We consider the minimization problem over a constrained domain associated to (3.1) and relax the constraint by introducing a penalty term in the minimized functional (see [20]). The added term is the following:

$$
\frac{1}{\varepsilon} \mathbb{D}(\mathbf{u}): \mathbb{D}(\mathbf{u})
$$

so that $\mathbb{D}(\mathbf{u})$ goes to zero when $\varepsilon$ goes to zero and $\mathbf{u}$ tends to a rigid motion in $B$.

The variational formulation obtained is:

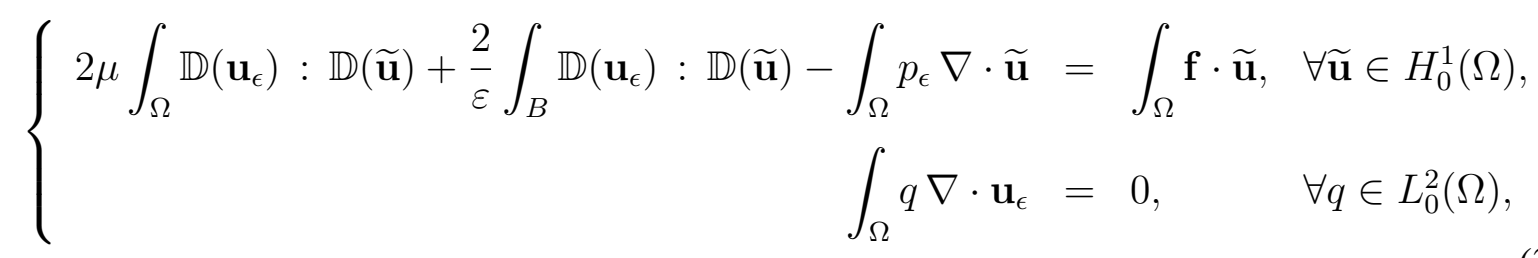


It has been proven in [16] that, in this framework, the penalty method converges linearly in $\varepsilon$ : the solution of problem (3.2) $\mathbf{u}_{\epsilon}$ converges to the solution $\mathbf{u}$ of problem (3.1) as $\varepsilon$ vanishes, and the convergence is of order 1 in $\varepsilon$. We refer to [23] for a detailed analysis of a scalar version of this problem, which provides an error estimate for the space-discretized problem at the order $\varepsilon+h^{1 / 2}$, where $h$ is the typical size of the space discretization.

\subsection{Variational formulation for the mesoscopic model}

The intermediate model described in Section 2.2 consists of the Stokes equations with a particular source term, and we use the following classical variational formulation:

$$
\left\{\begin{aligned}
2 \mu \int_{\Omega} \mathbb{D}(\mathbf{u}) \cdot \mathbb{D}(\widetilde{\mathbf{u}})-\int_{\Omega} p \nabla \cdot \widetilde{\mathbf{u}} & =\int_{\Omega} \mathbf{f} \cdot \widetilde{\mathbf{u}}, & & \forall \widetilde{\mathbf{u}} \in H_{0}^{1}(\Omega), \\
\int_{\Omega} q \nabla \cdot \mathbf{u} & =0, & & \forall q \in L_{0}^{2}(\Omega),
\end{aligned}\right.
$$

where

$$
\mathbf{f}=\sum_{i=1, \ldots, N} m \mathbf{g} \chi_{b}^{i}
$$

Note that, for regularity reasons, the delta function in (2.6) has been replaced by the characteristic function associated to a non-zero volume representing the swimmer.

\subsection{Contact handling}

As mentioned previously, if we consider particles with smooth surfaces in a Stokes flow, there should be no contact between them. Yet in actual simulations, collisions between particles are likely to occur because of the discretization in time. From a numerical point of view, it means that the particles may overlap when their positions are updated after the velocity field computation. The treatment of possible overlaps is even more important in the case of dense suspensions, if we want to avoid small time steps. For that purpose we use a numerical method proposed in [22], where inelastic collisions between rigid particles are computed. It consists of projecting the velocity field onto some convex admissible set depending on the current configuration, so that particles do not overlap.

Let us detail the method in the case of spherical particles: we denote by $\mathbf{X}^{n}:=\left(\mathbf{x}_{i}^{n}\right)_{i=1, \ldots, N}$ the position of $N$ particles (more precisely, the position of their gravity centre) at time $t_{n}$, and by $\hat{\mathbf{V}}^{n}$ their a priori velocity. As stated before, the a priori updated position of the particles, defined as

$$
\mathbf{X}^{n+1}=\mathbf{X}^{n}+\Delta t \cdot \hat{\mathbf{V}}^{n},
$$

may lead to non-admissible configuration, in the sense that the particles overlap. To avoid this, we project the velocities onto the following set

$$
K\left(\mathbf{X}^{n}\right)=\left\{\mathbf{V} \in \mathbb{R}^{2 N}, D_{i j}\left(\mathbf{X}^{n}\right)+\Delta t \mathbf{G}_{i j}\left(\mathbf{X}^{n}\right) \cdot \mathbf{V} \geq 0, \forall i<j\right\},
$$


where

$$
D_{i j}\left(\mathbf{X}^{n}\right)=\left|\mathbf{x}_{i}^{n}-\mathbf{x}_{j}^{n}\right|-2 R_{b}
$$

denotes the signed distance between two spheres $B_{i}$ and $B_{j}$ and

$$
\mathbf{G}_{i j}\left(\mathbf{X}^{n}\right)=\nabla D_{i j}=\left(\ldots, 0,-\mathbf{e}_{i j}, 0, \ldots, 0, \mathbf{e}_{i j}, 0, \ldots\right), \quad \mathbf{e}_{i j}=\frac{\mathbf{x}_{j}-\mathbf{x}_{i}}{\left|\mathbf{x}_{j}-\mathbf{x}_{i}\right|}
$$

is the gradient of the distance.

Remark 1. Denote $E\left(\mathbf{X}^{n}\right)=\left\{\mathbf{V} \in \mathbb{R}^{2 N}, D_{i j}\left(\mathbf{X}^{n}+\Delta t \mathbf{V}\right) \geq 0, \forall i<j\right\}$ the set of velocity fields $\mathrm{V}$ such that particles, at position $\mathrm{X}^{n}$ at time $t_{n}$ and with velocity $\mathrm{V}$, do not overlap at the next time step. The constraint $D_{i j}\left(\mathbf{X}^{n}\right)+\Delta t \mathbf{G}_{i j}\left(\mathbf{X}^{n}\right) \cdot \mathbf{V} \geq 0$ is the linearized form of the constraint $D_{i j}\left(\mathbf{X}^{n}+\Delta t \mathbf{V}\right) \geq 0$ and, furthermore, it can be shown that $K\left(\mathbf{X}^{n}\right) \subset E\left(\mathbf{X}^{n}\right)$. It means in particular that particles with admissible velocities at time $t_{n}$ do not overlap at time $t_{n+1}$.

In order to avoid overlapping, the following splitting procedure is proposed: in a first step, we solve the variational problem without taking into account the possible overlapping of the particles (thus defining the a priori velocity of the spheres), then compute the projection of this a priori velocity onto the set of admissible velocities defined by (3.4). The constrained problem is formulated as a saddle-point problem, by using the introduction of Lagrange multipliers:

$$
\left\{\begin{array}{l}
\text { Find }\left(\mathbf{V}^{n}, \boldsymbol{\Lambda}^{n}\right) \in \mathbb{R}^{2 N} \times \mathbb{R}_{+}^{N(N-1) / 2} \text { such that } \\
\mathcal{J}\left(\mathbf{V}^{n}, \lambda\right) \leq \mathcal{J}\left(\mathbf{V}^{n}, \boldsymbol{\Lambda}^{n}\right) \leq \mathcal{J}\left(\mathbf{V}, \mathbf{\Lambda}^{n}\right), \quad \forall(\mathbf{V}, \lambda) \in \mathbb{R}^{2 N} \times \mathbb{R}_{+}^{N(N-1) / 2},
\end{array}\right.
$$

with the following functional:

$$
\mathcal{J}(\mathbf{V}, \lambda)=\frac{1}{2}\left|\mathbf{V}-\hat{\mathbf{V}}^{n}\right|^{2}-\sum_{1 \leq i<j \leq N} \lambda_{i j}\left(D_{i j}\left(\mathbf{X}^{n}\right)+\Delta t \mathbf{G}_{i j}\left(\mathbf{X}^{n}\right) \cdot \mathbf{V}\right) .
$$

Notice that the number of Lagrange multipliers corresponds to the number of possible contacts. In particular, if there is no contact between particles $i$ and $j$, then $\Lambda_{i j}=0$ and the Lagrange multiplier is not activated; conversely, if there is a contact between the two spheres, then $\Lambda_{i j}$ may be positive and the corresponding auxiliary field allows the velocity field to satisfy the nooverlapping constraint. The approximate reaction fields $\Lambda^{n}=\left(\Lambda_{i j}^{n}\right)$ is the dual component of a solution to the associated saddle-point problem. This problem is solved by an Uzawa algorithm (see, e.g., [4]).

The interest in the procedure relies on the possibility to use any suitable solver for the computation of the dynamics. Contacts are handled at a second stage, without any consideration of the proper dynamics. At some point, it allows the use of any solver for the resolution of the dynamics problem: then the so-called predicted velocity field is projected onto the set of admissible velocity fields.

Remark 2. In the mesoscopic model, the bacterial bodies are modelled by spheres, and we apply the contact algorithm just described in order to prevent their overlapping. In the fully microscopic 
model, the bacterial bodies are modelled as elongated ellipses. In order to handle contacts, one should adapt the algorithm to the case of ellipses; that involves computing the distance between two elliptic bodies, which is not straightforward. In order to simplify computations, we rather handle contacts between the smallest spheres containing the ellipses.

\subsection{Discrete tumbling}

As described in 2.3, the chemotactic behaviour of the bacteria is modelled as a Poisson process with variable intensity (the frequency of tumbling $\lambda$ ). We time-discretise this process as follows: let $\Delta t_{\text {tumb }}>0$ be a fixed small time-step, $P_{\text {tumb }}$ is the probability of a swimmer to tumble at least one time during $\Delta t_{\text {tumb }}$. This probability can be computed and depends on the values of $\Delta t_{t u m b}$ and $\lambda$ :

$$
P_{\text {tumb }}\left(\lambda, \Delta t_{\text {tumb }}\right)=1-\exp \left(-\lambda \Delta t_{\text {tumb }}\right) \sim \lambda \Delta t_{\text {tumb }} .
$$

Now, for each bacterium, the frequency of tumbling $\lambda$ varies in time following (2.8). We discretize the lagrangian derivative of the oxygen concentration $\mathrm{D} c / \mathrm{D} t$ evaluated by the $i$-th bacterium along its trajectory as follows:

$$
\frac{\mathrm{D} c}{\mathrm{D} t}(t)_{i} \approx \frac{c(t)_{i}-c\left(t-\Delta t_{\text {tumb }}\right)_{i}}{\Delta t_{\text {tumb }}},
$$

where $c(t)_{i}$ denotes the oxygen concentration at time $t$, at the position occupied by the $i$-th bacterium at time $t$.

Thus the value of $P_{\text {tumb }}$ also varies in time, depending on the sign of the discrete lagrangian derivative. At each time $t^{m}=m \Delta t_{t u m b}$, bacterium $i$ performs a random reorientation (a tumble) with probability:

$$
\left(P_{\text {tumb }}\right)_{i}^{m} \sim \lambda_{0}\left(1-\varepsilon \phi\left(c\left(t^{m}\right)_{i}-c\left(t^{m}-\Delta t_{\text {tumb }}\right)_{i}\right)\right) \Delta t_{\text {tumb }},
$$

where we recall that $\phi$ is the sign function. Note that with this procedure we neglect the fact that there can be more than one reorientation during $\Delta t_{t u m b}$. But this has no influence on the result as long as we neglect correlation between the directions before and after tumbling.

In the simulations presented in the next section, we have used the following values for the parameters of the tumbling model : $\epsilon=1$ and $\lambda_{0} \Delta t_{\text {tumb }}=0.5$. Note that this amounts to use a tumbling probability of $P_{t u m b}=0$ if the direction is favorable, and $P_{t u m b}=1$ if it is not. More realistic simulations can be performed by taking a smaller value of $\epsilon$ and of $\lambda_{0} \Delta t_{t u m b}$, which can be interpreted as the ratio between the time-step $\Delta t_{\text {tumb }}$ used to discretize the Poisson process and the typical tumbling period $1 / \lambda_{0}$.

\section{Numerical results}

We have simulated individual and collective dynamics in moderate to dense bacterial suspensions, using both models described in Section 2. The results in Sections 4.1 and 4.2 have been obtained 
with the fully microscopic model, whereas for the results in Section 4.3 we have used the mesoscopic model. In Section 4.1 we analyse the instantaneous velocity field obtained around one bacterium in the absence of interactions, and after that we focus on the simulation of hydrodynamic interactions: between a bacterium and a wall, and between two and several bacteria. Then, we show the velocity field obtained in moderate to dense suspensions. In Section 4.2, we concentrate on the effect of chemotaxis in a moderately concentrated suspension using the microscopic model coupled with the model for chemotaxis. Finally, Section 4.3 is devoted to the simulation of bioconvective dynamics in dense suspensions using the mesoscopic model described in 2.2.

Numerical results have been obtained with data provided in Tables 1 and 2.

\begin{tabular}{|c|c|c|c|c|c|}
\hline Figures & $2,3,5,7$ & 4 & 6 & 8 & 9 \\
\hline Domain & unit square & $i d$ & $i d$. & $i d$. & id. \\
\hline \multicolumn{6}{|l|}{ Mesh } \\
\hline Vertices & 26765 & 47646 & 47646 & 26765 & 26765 \\
\hline Triangles & 52928 & 94490 & 94490 & 52928 & 52928 \\
\hline \multicolumn{6}{|l|}{ Bacteria } \\
\hline Number of individuals & $1 \sim 5$ & 1 & 2 & 100 & 1000 \\
\hline Head semi-major axis $* 10^{3}$ & 54.0 & 27.0 & 20.0 & 20.0 & 14.0 \\
\hline Head semi-minor axis $* 10^{3}$ & 20.0 & 10.0 & 7.0 & 7.0 & 7.0 \\
\hline Flagellum semi-major axis $* 10^{3}$ & 80.0 & 54.0 & 40.0 & 40.0 & 27.0 \\
\hline Flagellum semi-minor axis $* 10^{3}$ & 7.0 & 3.5 & 3.5 & 3.5 & 3.5 \\
\hline Distance head-flagellum $* 10^{3}$ & 150.0 & 95.0 & 75.0 & 75.0 & 55.0 \\
\hline Propulsion intensity & 13.5 & 6.5 & 1.0 & 1.0 & 1.0 \\
\hline \multicolumn{6}{|l|}{ Fluid boundary conditions } \\
\hline Upper / lower walls & $\mathbf{u}=\mathbf{0}$ & $i d$. & $i d$. & $i d$. & $i d$. \\
\hline Lateral walls & periodicity & id. & $i d$. & $i d$. & $i d$. \\
\hline
\end{tabular}

Table 1: Parameters for the numerical simulations (I): hydrodynamic interactions.

\subsection{Simulation of individual and collective motion with the complete model}

\section{Velocity field induced by a single bacterium}

As pointed out previously, the key feature in the dynamics of a single bacterium is that the net propulsive force of the flagella must equal the opposing drag force of the body connected to the flagella. Thus, Fig. 2 (t) describes the velocity fluid flow generated by a single bacterium and the contour lines of the velocity. The contours reveal the extent of the fluid disturbance created 


\begin{tabular}{|c|c|c|c|}
\hline Figures & 10 & 11 & 12,13 \\
\hline Domain & square & rectangle & drop \\
\hline (max.) width & 1.00 & 2.00 & 1.10 \\
\hline (max.) height & 1.00 & 1.00 & 0.25 \\
\hline \multicolumn{4}{|l|}{ Mesh } \\
\hline Vertices & 26765 & 23159 & 10516 \\
\hline Triangles & 52928 & 45716 & 20530 \\
\hline \multicolumn{4}{|l|}{ Bacteria } \\
\hline Number of individuals & 100 & 1500 & 1500 \\
\hline Head semi-major axis $* 10^{3}$ & 13.5 & 10.0 & 5.0 \\
\hline Head semi-minor axis $* 10^{3}$ & 7.0 & 10.0 & 5.0 \\
\hline Flagellum semi-major axis $* 10^{3}$ & 27.0 & - & - \\
\hline Flagellum semi-minor axis $* 10^{3}$ & 3.5 & - & - \\
\hline Distance head-flagellum $* 10^{3}$ & 55.0 & - & - \\
\hline Propulsion intensity $* 10^{3}$ & 250.0 & 100.0 & 50.0 \\
\hline \multicolumn{4}{|l|}{ Fluid boundary conditions } \\
\hline Upper wall & $\mathbf{u}=\mathbf{0}$ & $i d$. & id. \\
\hline Lower wall & $\mathbf{u}=\mathbf{0}$ & $i d$. & $i d$. \\
\hline Lateral walls & periodicity & periodicity & $\mathbf{u}=\mathbf{0}$ \\
\hline \multicolumn{4}{|l|}{ Oxygen concentration } \\
\hline Initial condition & $c \equiv 1$ & $c \equiv 1$ & $c \equiv 1$ \\
\hline Lateral boundary condition & periodicity & periodicity & $c=1$ \\
\hline Upper boundary condition & $c=1$ & $c=1$ & $c=1$ \\
\hline Lower boundary condition & $\nabla c \cdot \mathbf{n}=0$ & $\nabla c \cdot \mathbf{n}=0$ & $\nabla c \cdot \mathbf{n}=0$ \\
\hline Diffusion parameter & 0.20 & 0.50 & 0.01 \\
\hline Volumic consumption rate & 110.00 & 6.50 & 4.70 \\
\hline Propulsion modulation function & $c$ & $\min (1, c / 0.25)$ & $\min (1, c / 0.25)$ \\
\hline Time step & $2.5 \cdot 10^{-2}$ & $5.0 \cdot 10^{-2}$ & $5.0 \cdot 10^{-2}$ \\
\hline
\end{tabular}

Table 2: Parameters for the numerical simulations (II): chemotactic results. 
by the bacterium. Besides, Fig. 2 (b) provides the streamlines of the instantaneous velocity field, revealing recirculations patterns.

\section{Interaction of a bacterium with a wall}

We address the swimming of individual bacteria near surfaces. Although the specific interactions between helical flagella and a boundary are crucial, our model does not focus on such a complex description of the helical flagella, but aims at describing some phenomenological features. Fig. 3 describes the velocity fluid flow generated by a bacterium placed close to a boundary, and the contour lines around it. Fig. 4 reproduces the trajectory of a bacterium which is initially located with an angle $\pi / 4$ with respect to the boundary ; the motion of the self-propelled bacterium illustrates the reflection to the wall. Note that, from a numerical point of view, the flagella may be located outside the domain: the propulsive force is simply spread over the part of the flagellum which is located inside the domain, thus preserving global force balance (of course, the flagellum should not be entirely located outside the domain).

\section{Pairwise interactions}

Consider two bacteria next to each other and prescribed to move in a parallel direction. The flow patterns shown on Fig. 5 suggest that the flow tends to push the bacteria toward each other, as there is a component of the fluid flow pointing into the direction of the neighbouring bacterium, thus revealing the attraction of the fluid flow. We emphasize that Fig. 6 evidences a behaviour which has been described in [12] and in [19]: trajectories of initially parallel bacteria in the mirror image configuration attract and rotate outward. Then when rotated sufficiently outward, the bacteria swim off. Note that the flagella may overlap in our case, as they only model the phenomenological response of the flagella over the fluid.

\section{Interactions between five ellipsoidal bacteria}

Fig. 7 shows the velocity fluid flow and streamlines around five bacteria. The velocity distribution shows the attraction of most of the cells to each other. Transverse flows between the body of a follower and the tail of a preceder can be observed. As pointed out in [5], the numerical results show that there is little front-to-back penetration of fluid. The exchange is almost lateral: the leading heads push water forward whereas the tail-end cells push water backward, generating much of the collective forward propulsion. Thus the associated interior flows are weak, but vortical regions can significantly enhance transport of suspended particles.

\section{Motion in moderate to dense suspensions}

Fig. 8 shows a moderate suspension including 100 bacteria, what represents a volumic concentration of $4.25 \%$. In this configuration, the velocity of a single bacterium (in absence of other swimmers) is of magnitude 2.5 times the length of the bacterium per second. In the suspension, the maximal fluid velocity is about 15 times the length of the bacteria per second: the velocity of 

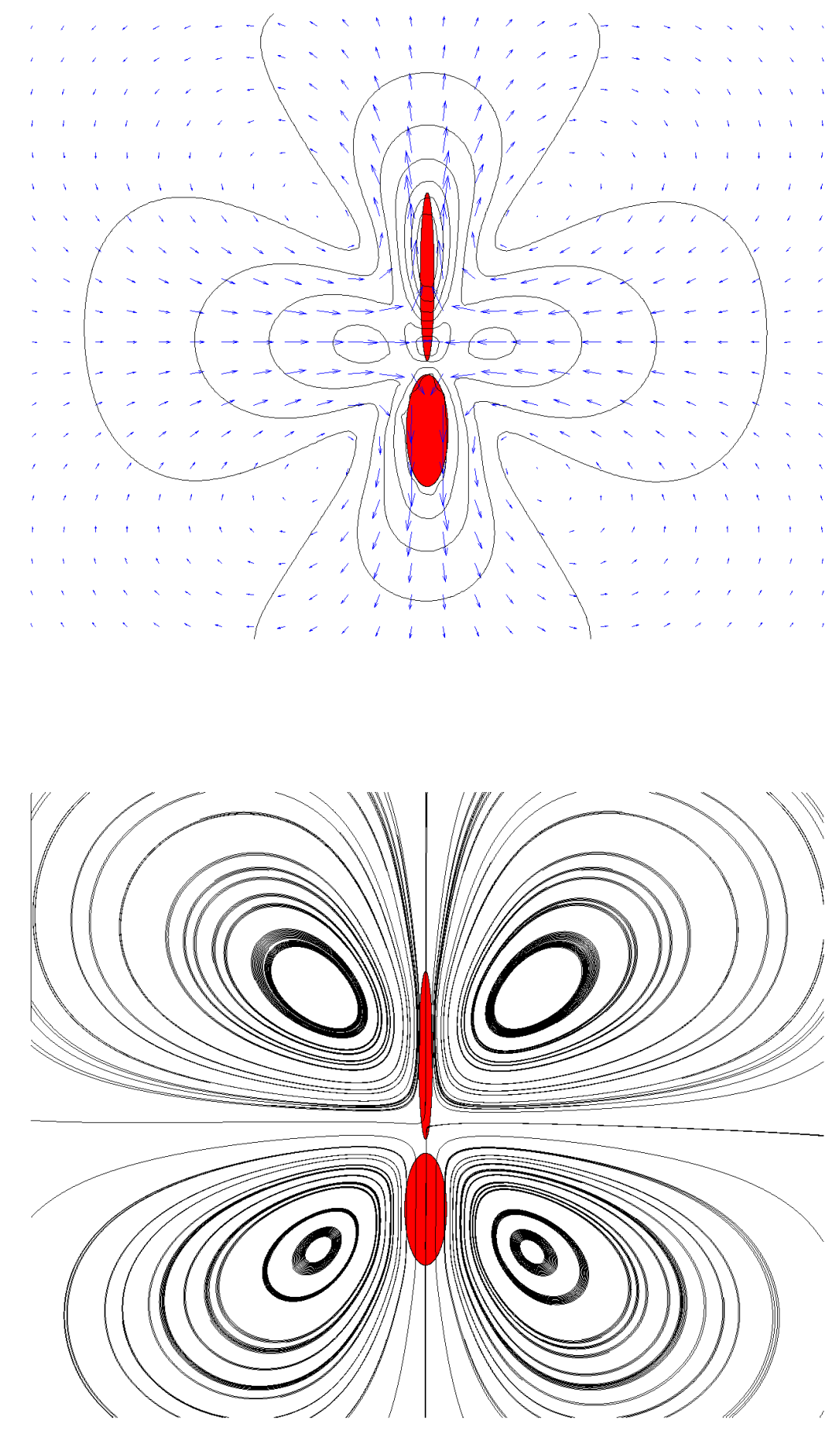

Figure 2: Velocity profiles ( (t) velocity field (b) streamlines ) in the fluid flow in the neighbourhood of an ellipsoidal bacterium 

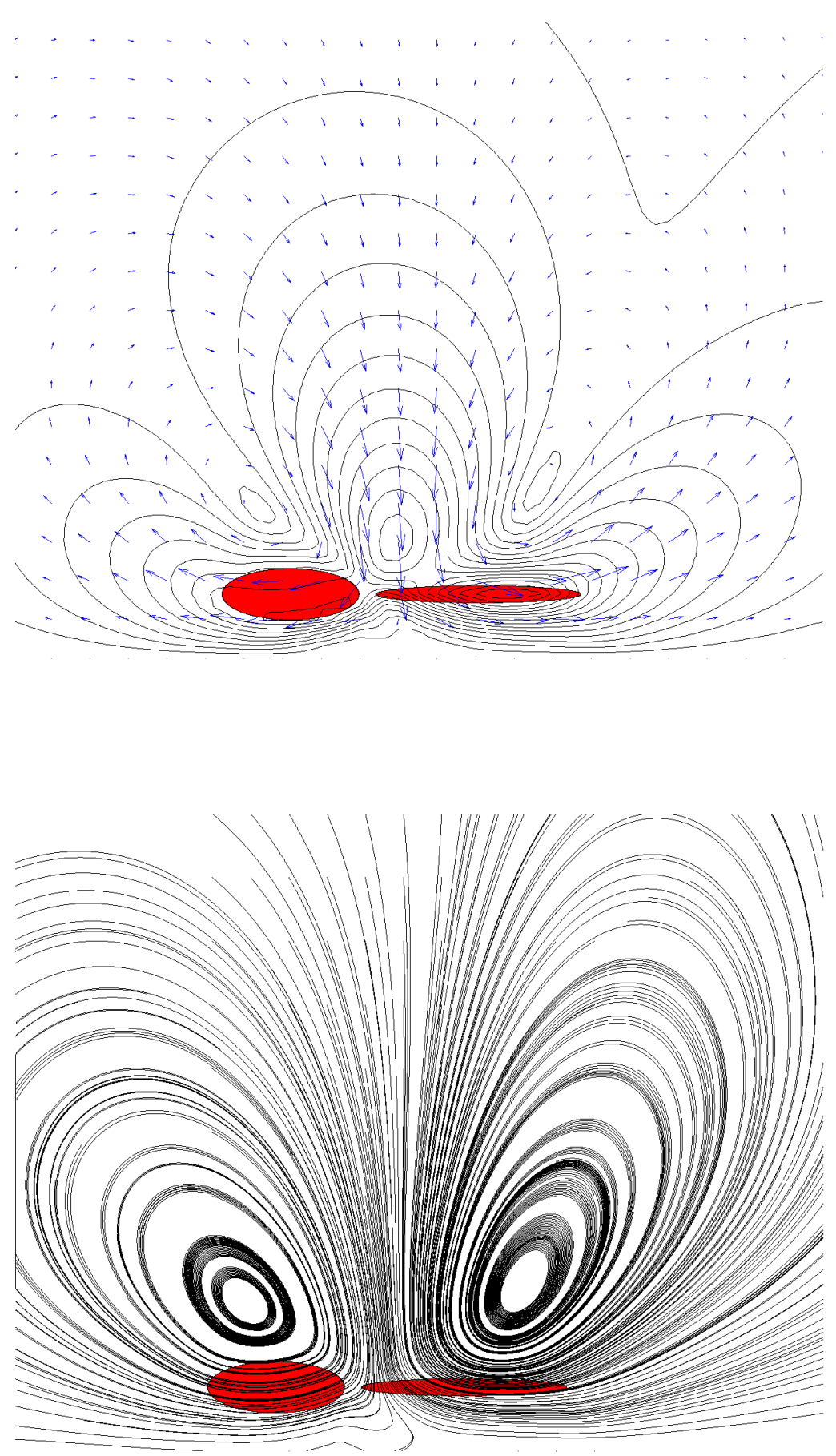

Figure 3: Velocity profiles ((t) velocity field (b) streamlines) in the fluid flow in the neighbourhood of an ellipsoidal bacterium located near a solid wall 


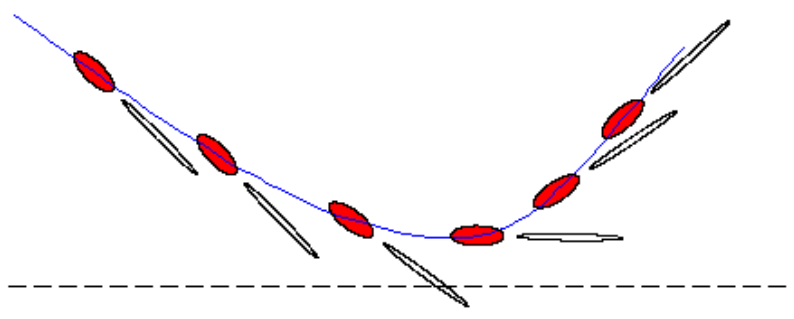

Figure 4: Trajectory of an ellipsoidal bacterium moving towards a rigid wall

the flow is thus signicantly enhanced by collective dynamics, as observed in experimental studies (see [31] and [5]). This feature is even stronger in simulations of dense suspensions: Fig. 9 shows a dense suspension including 1000 bacteria, what represents a volumic concentration of $28 \%$. In this case, the maximal fluid velocity reaches 25 times the length of the bacterium per second, consistent with the experimental study in [31], which has revealed collective flow speeds that exceed the speed of the individual bacterium by an order of magnitude. We also emphasize that the microscopic model is able to reproduce the low turbulence dynamics observed in dense suspensions (see $[5,36])$.

\subsection{Chemotactic behaviour}

Bacteria may require oxygen for swimming. In this case, chemotactic behaviour based on run and tumble cycles allows them to swim up the oxygen gradient on the long time scale. Meanwhile, oxygen transport into the bacterial suspension from the adjacent air involves molecular diffusion and also advection by collectively generated streaming. Fig. 10 qualitatively reproduces this main feature of chemotactic response linked to hydrodynamic interactions. We may observe screenshots of the oxygen distribution and position of the microstructure (100 bacteria) at different time steps in a square domain, for which the upper wall represents the air-fluid interface. At initial time, the oxygen distribution is homogeneous in space and the bacteria have been placed randomly in the domain. After one time step, we observe a local consumption of oxygen, which is larger where the density of bacteria is high. After 20-50 time steps, an oxygen gradient has appeared through the conjunction of $i$ ) consumption of oxygen by the bacteria, $i i$ ) oxygen supply from the fluid-air interface and iii) diffusion of oxygen into the whole domain. After 1000 time steps, most of the bacteria have migrated towards the supply boundary, following the oxygen gradient. Notice also that, after 3400 time steps, the bacteria are still mainly located at the fluid-air interface and, besides, the oxygen concentration far from the supply boundary has increased because of the low density of bacteria. From a numerical point of view, numerical data, such as diffusion parameter of oxygen in water and rate of oxygen consumption by the bacteria, have been fitted in order to reproduce "just in time" oxygen delivery (as mentioned in [5]). Note that gravity effects have been neglected in this experiment: the bacteria motion is driven by the run and tumble dynamics governed by the 

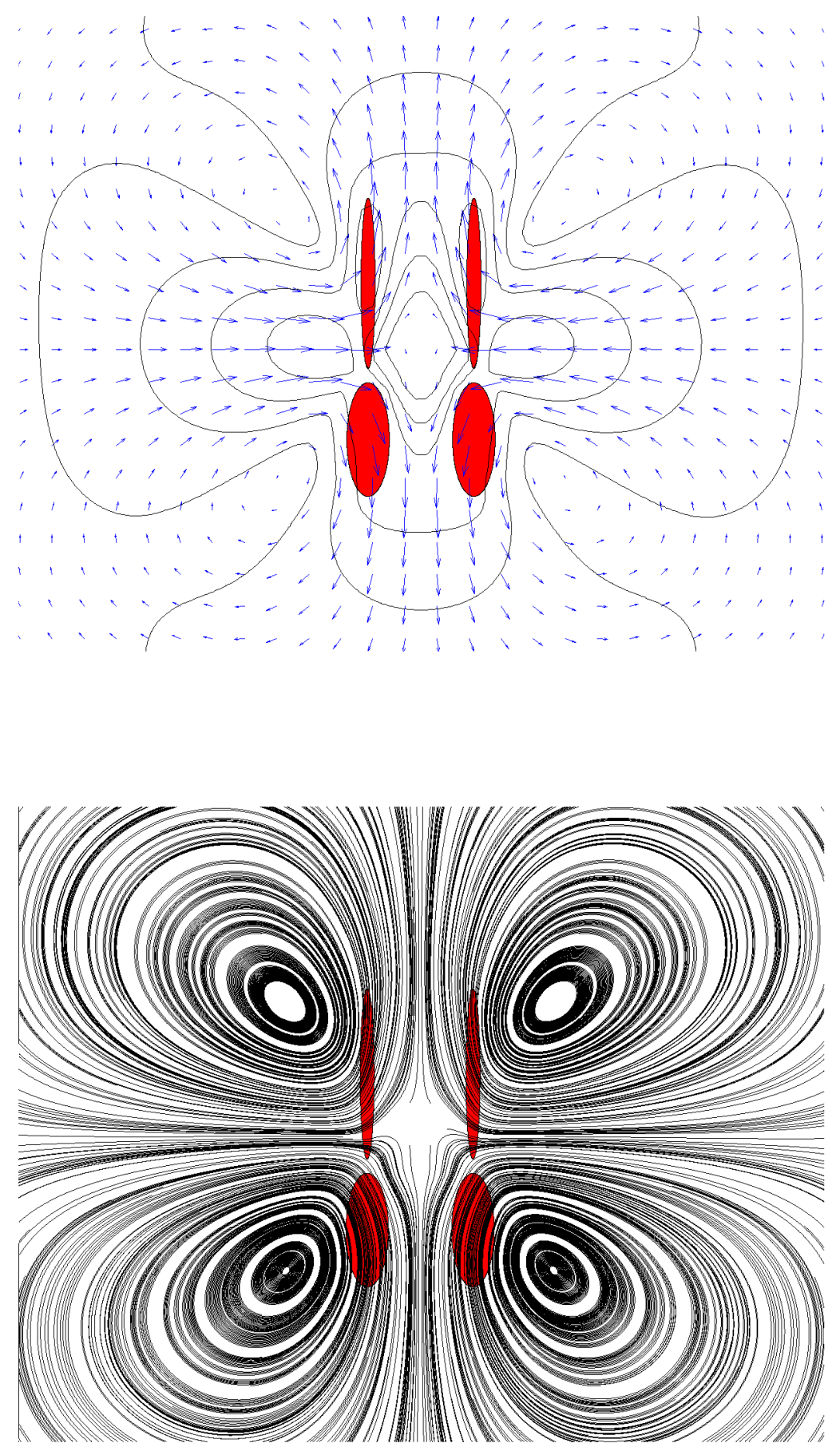

Figure 5: Velocity profiles ((t) velocity field (b) streamlines) in the fluid flow in the neighbourhood of two ellipsoidal bacteria interacting through the fluid flow 

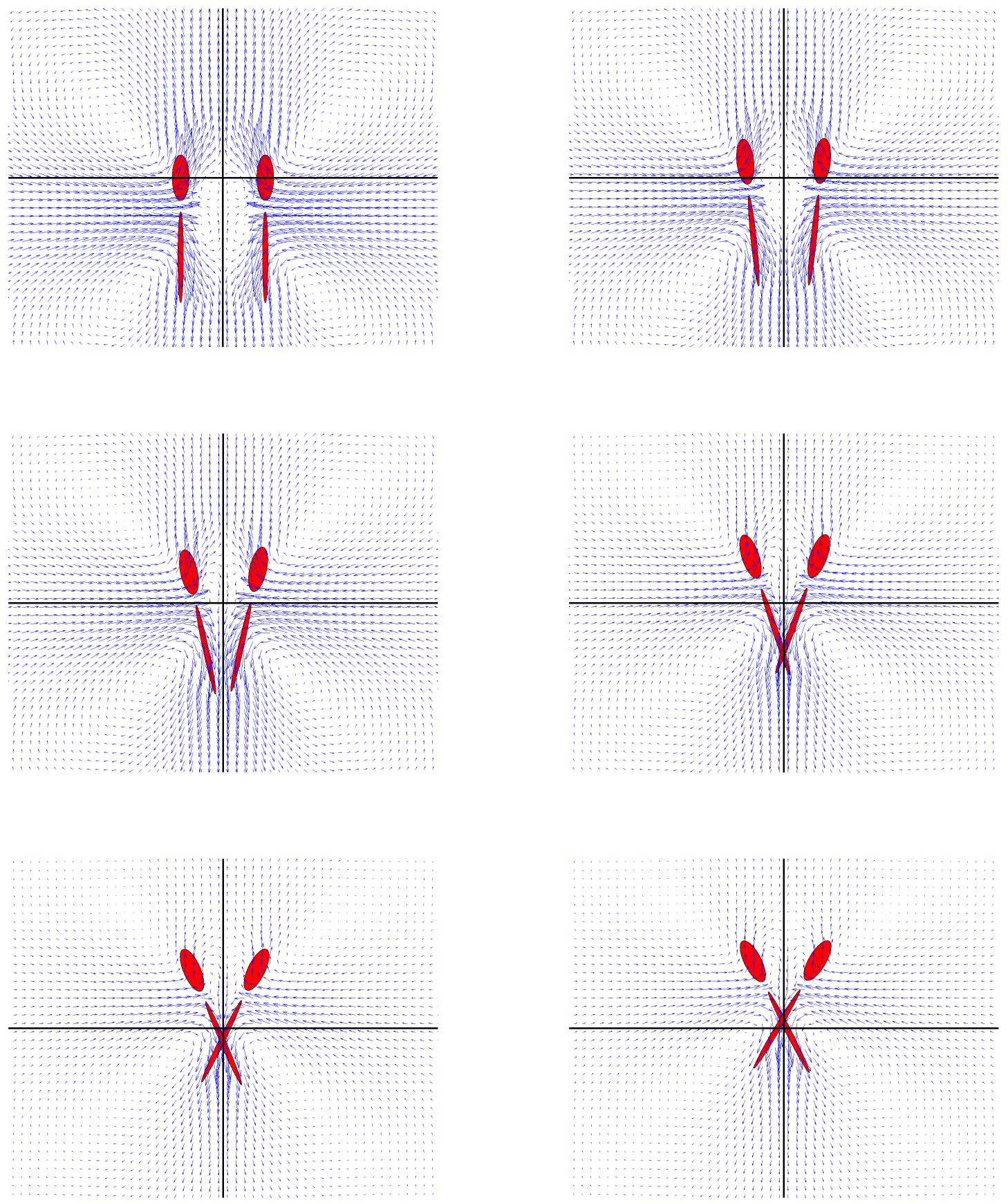

Figure 6: Hydrodynamic interactions between two close bacteria 

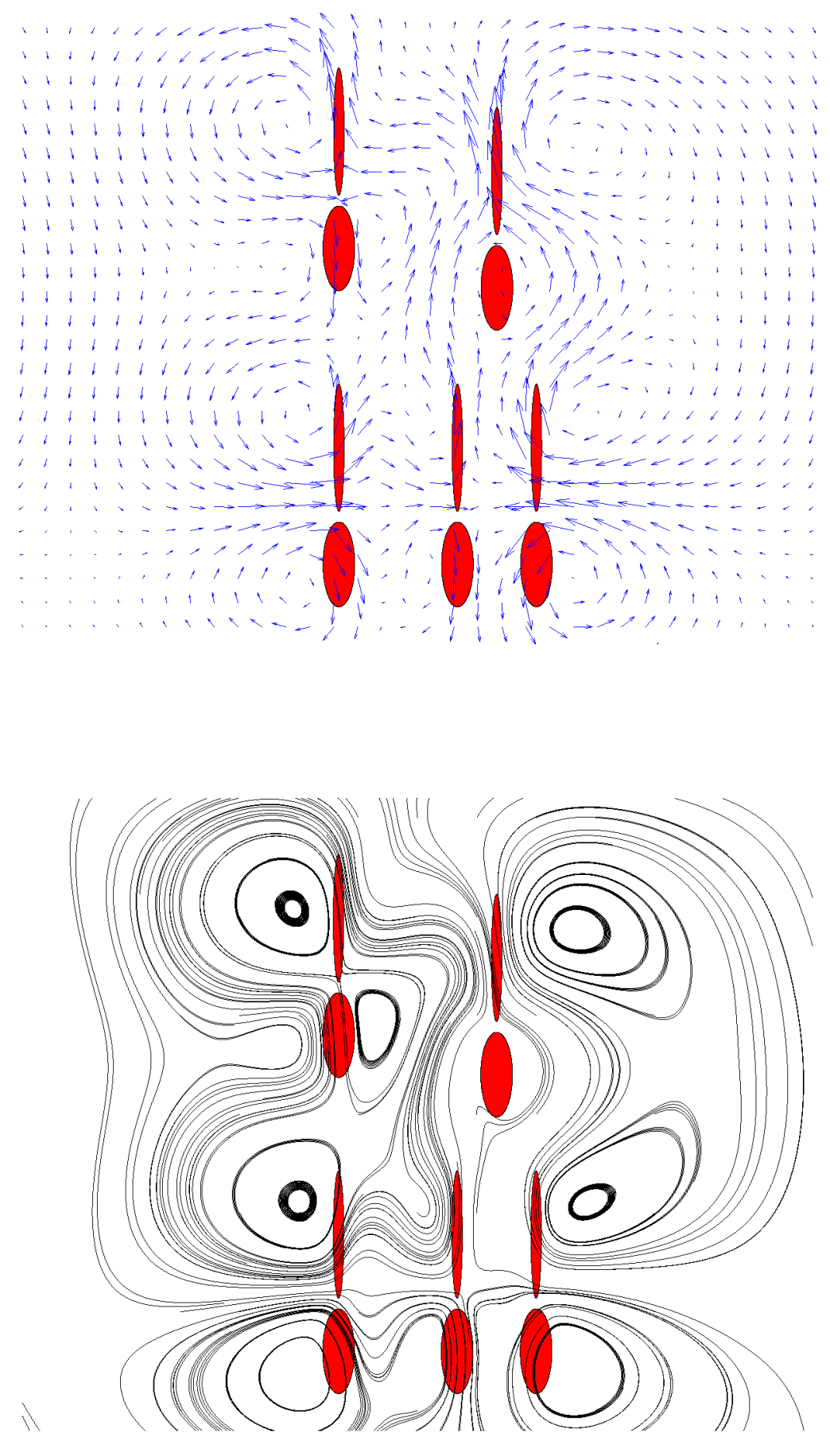

Figure 7: Velocity profiles ((t) velocity field (b) streamlines) in the fluid flow in the neighbourhood of five ellipsoidal bacteria interacting through the fluid flow 


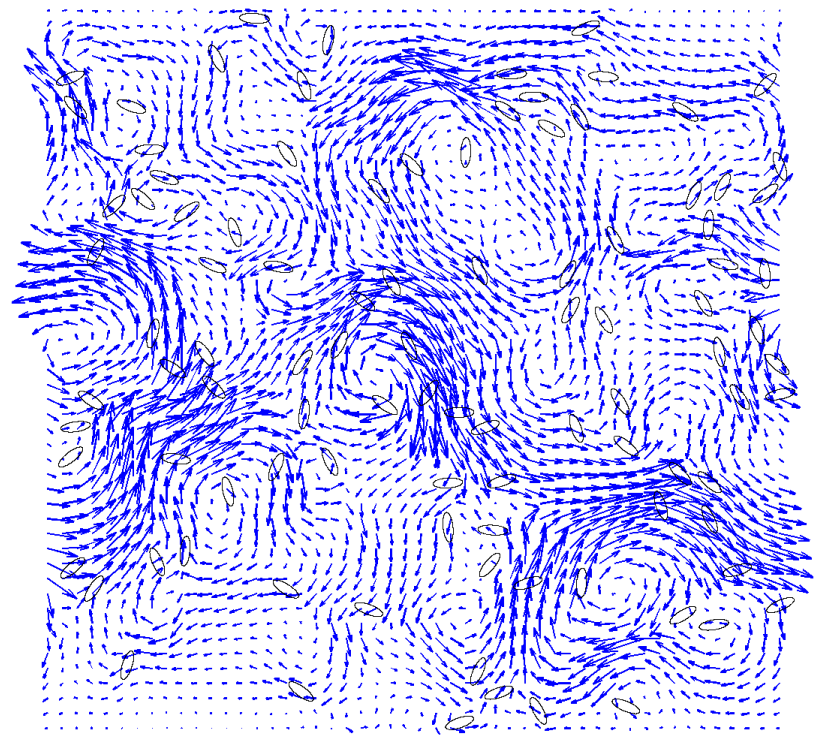

Figure 8: Moderate suspension of 100 ellipsoidal bacteria in a flow at rest, at a given time step

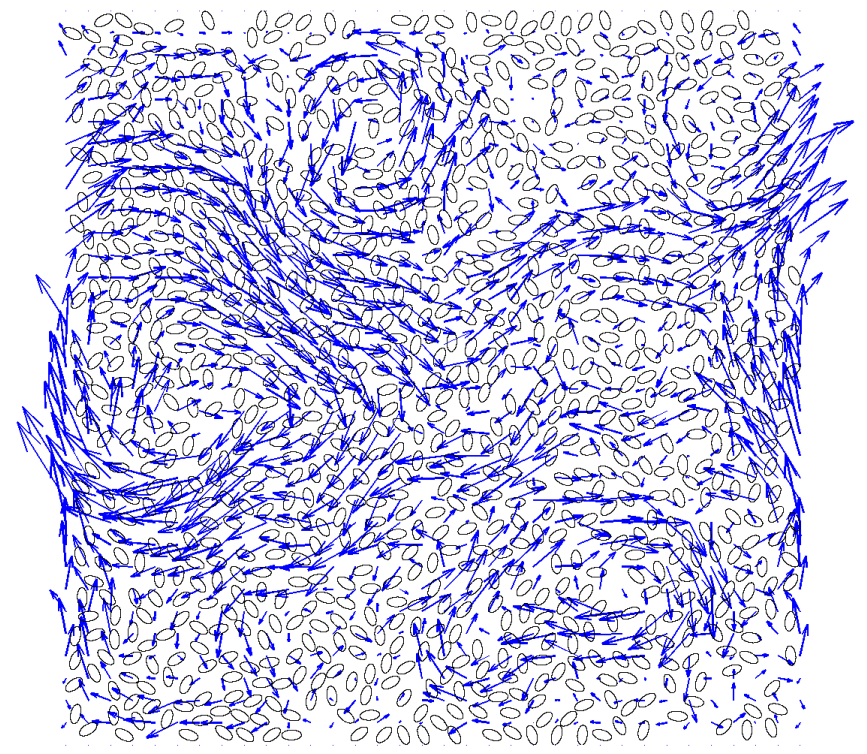

Figure 9: Dense suspension of 1000 ellipsoidal bacteria in a flow at rest, at a given time step 
fluid flow and oxygen distribution, which also depends on the bacteria consumption.

\subsection{Simulation of bioconvective dynamics with the mesoscopic model cou- pled to chemotaxis}

The response to oxygen gradients can initiate a collective behavior that creates striking hydrodynamic flows. In particular, we aim at reproducing bioconvection patterns in bacterial suspensions as those described in [8] and [34] (see Section 1.2). Arising from upward oxygentaxis and downward gravitational forcing, these dynamics are explained through the analysis of oxygen diffusion and consumption, chemotaxis, buoyant forcing and viscous fluid dynamics.

We have first simulated bioconvective dynamics arising in a domain with a horizontal upperboundary representing the fluid-air interface (see Table 2, rectangular domain). Gravity is taken into account in the computation through a buoyant forcing term in the Stokes equation (see the description of the mesoscopic model in Section 2.2). Fig. 11 reproduces the main observable features of the simulation. It can be observed that, starting from a homogeneous configuration (see Fig. $11(\mathrm{t})$ ), respiration of the bacteria depletes dissolved oxygen in the fluid medium in the following way (see Fig. $11(\mathrm{~m})$ ): first, bacteria swim up the resultant gradient of oxygen concentration toward the air and, second, bacteria which are located at the oxygen low-concentration part (i.e. at the bottom) are trapped, because the propulsion force is directly related to the oxygen consumption which has become very poor. Then, since bacteria are denser than water, accumulation of bacteria at the interface results in an unstable gradient of mean fluid density, leading to the emergence of falling plumes of bacteria (see Fig. 11 (b)). Note that these results are consistent with the instability of bioconvection in fluids with a horizontal meniscus [25]. The instabilities lead to a highly concentrated population at the bottom of the domain. Besides, they play a major role in the renewal of oxygen uptake for bacteria which were trapped in depleted regions, as convection not only concerns the fluid flow which has strong influence on the bacteria but also concerns the oxygen transport towards lower concentration regions.

We have also simulated bioconvective dynamics in a drop-like geometry: the results are shown in Figs. 12 and 13. This simulation qualitatively reproduces the experiment presented in [8], in which the evolution of a $B$. subtilis suspension in a sessile drop has been observed. The domain that has been considered for the numerical simulation resembles the sessile drop in the following sense: the domain is composed of a lower flat boundary (width 1.10), an upper flat boundary (width 1.10) and two lateral curved boundaries connecting the flat walls. In this setting, the lateral walls and the upper wall represent the air-fluid interface. As in Fig. 11, numerical results presented on Figs. 12 and 13 show similar issues such as the formation of a depletion zone due to oxygen consumption and the emergence of falling plumes due to bacterial accumulation at the fluid-air interface. It has been observed numerically that these instabilities do not occur in the absence of a flat fluid-air interface. On the contrary, increasing the width of the flat interface leads to the formation of an increasing number of instability patterns. Besides, we observe the generation of a persistent hydrodynamic vortex near the three-phase contact line, as observed in the experiment in [8]. This vortex traps cells near the contact line (see in particular Fig. 13 (m)), but it also enhances the uptake of oxygen into the suspension through advection. 

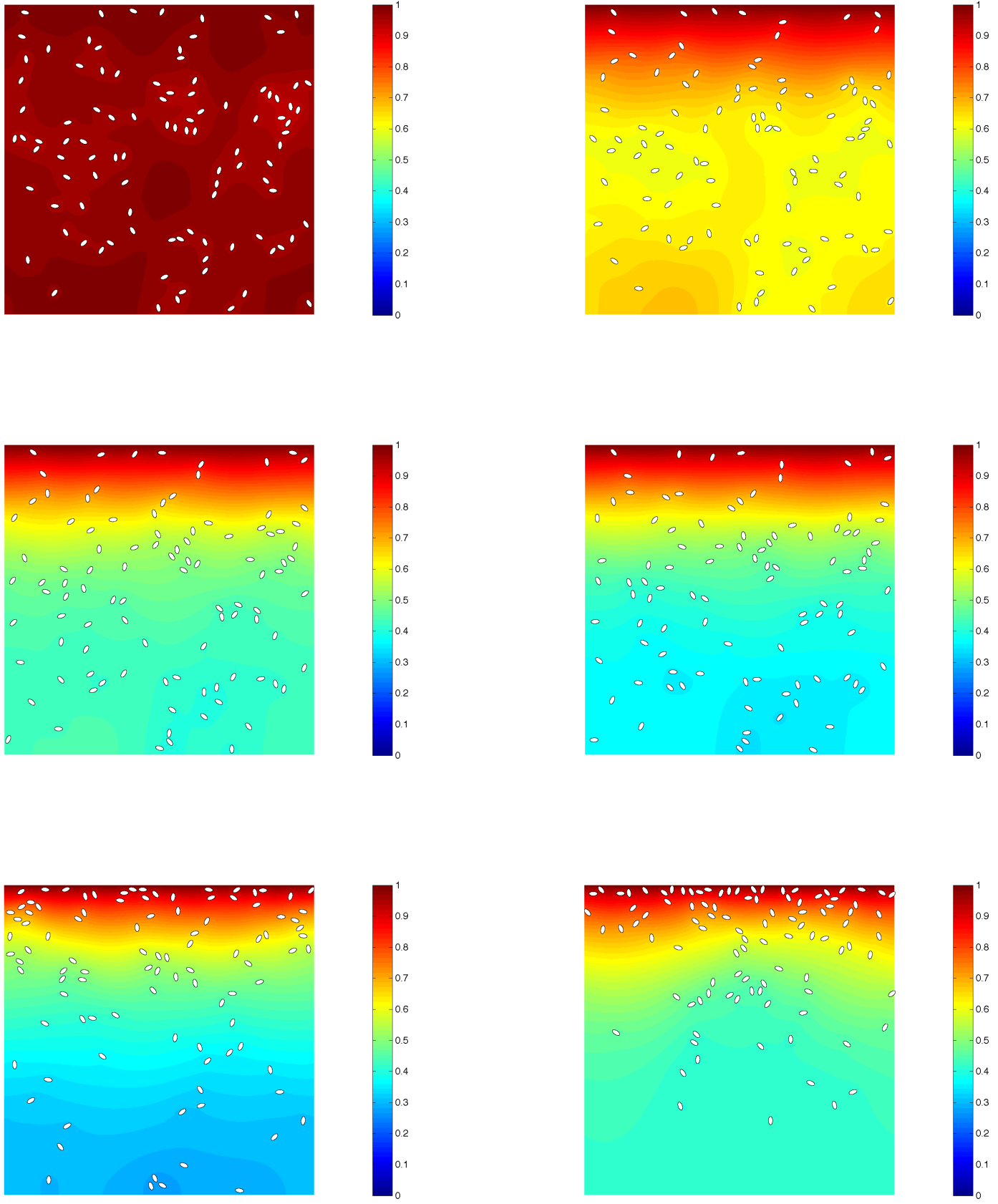

Figure 10: Position of 100 bacteria and oxygen distribution at different time steps $t_{n}=n \Delta t$. From left to right and from top to bottom : $n=1,10,20,40,50,1000,3400$. 

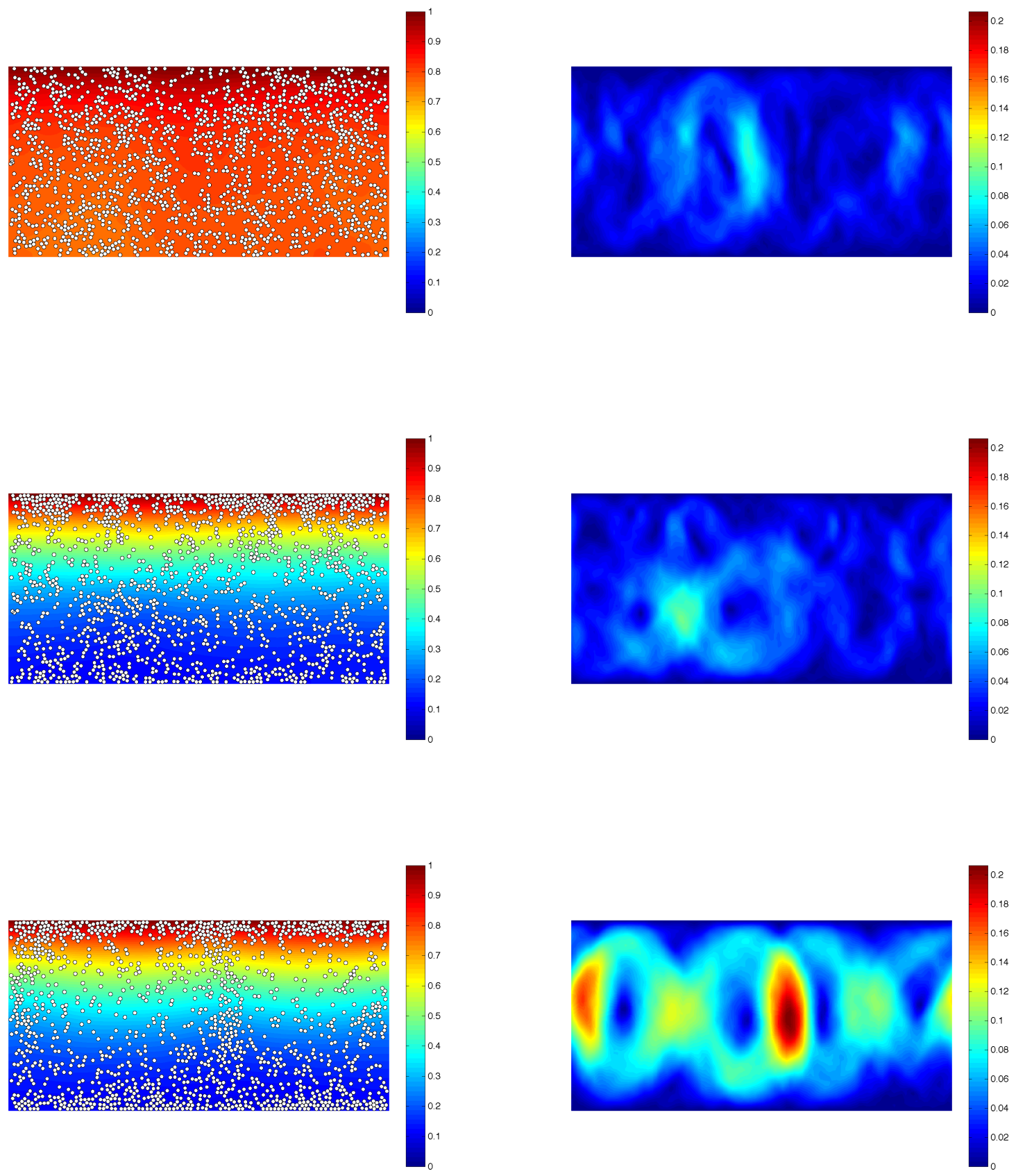

Figure 11: Dense suspension and oxygen distribution in a rectangular domain (1) and modulus of the velocity fluid (r) at different time steps $t_{n}=n \Delta t$. From top to bottom $n=3,99,1231$. 

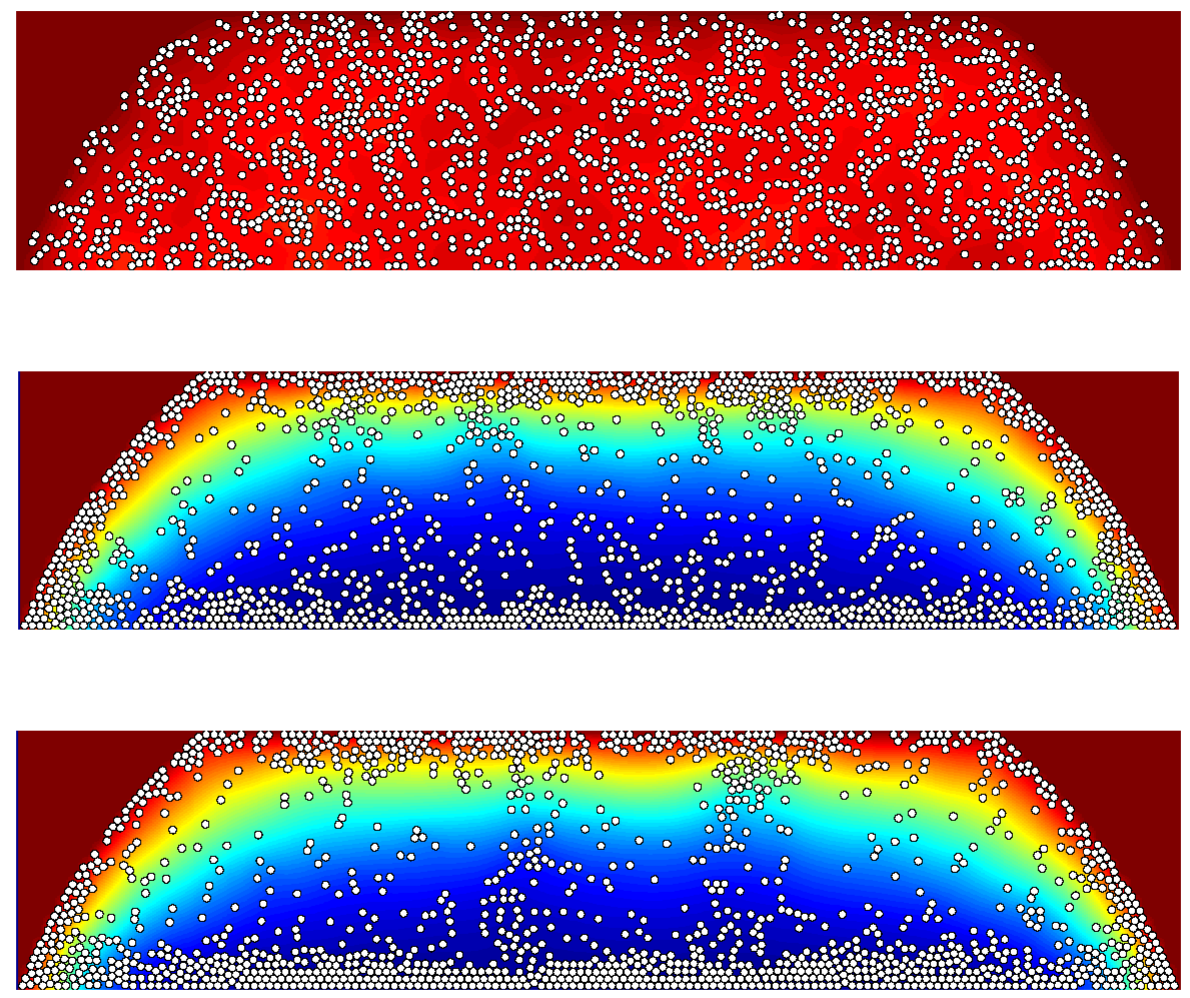

Figure 12: Dense suspension and oxygen distribution in a drop domain at different time steps $t_{n}=n \Delta t$. From top to bottom : $n=3,200,350$. 

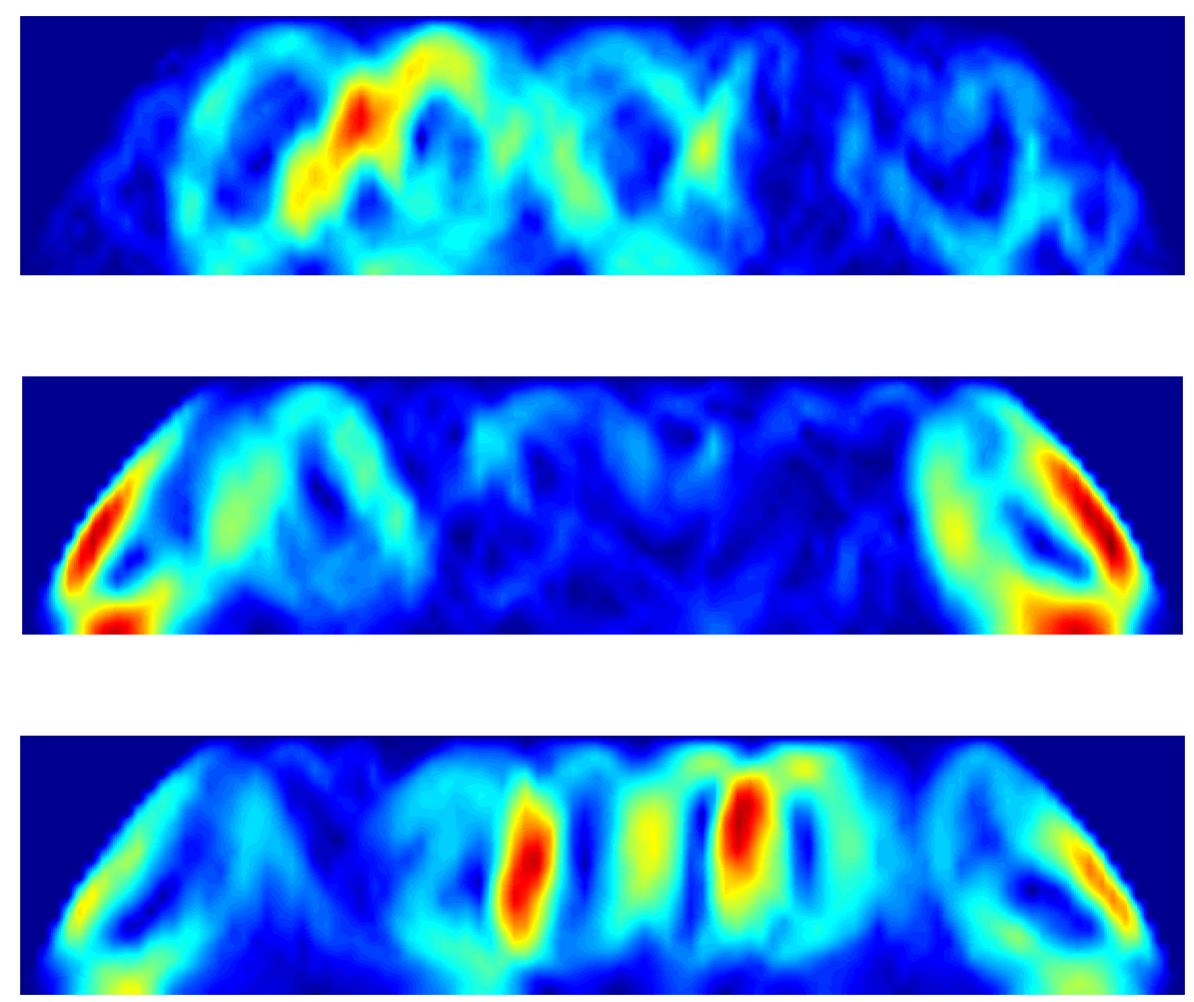

Figure 13: Modulus of the fluid velocity for a dense suspension in a drop domain at different time steps $t_{n}=n \cdot \Delta t$, for $n=3,200,350$ 


\section{Conclusion}

The fully microscopic model presented in Section 2.1 has proved to achieve a qualitatively accurate description of the fluid motion and hydrodynamic interactions. In order to reproduce the low-turbulence motion field in a suspension, or to compute the effective viscosity, one needs to solve accurately hydrodynamic interactions, and therefore the fully microscopic model should be used. But when focusing on bioconvection phenomena, the mesoscopic model (Section 2.2), combined with the simple stochastic model for chemotaxis (Section 2.3), is sufficient to reproduce the dynamics described in Section 1.2.

\section{References}

[1] H.C. Berg. Random walks in biology. Princeton University Press, Princeton, 1983.

[2] H.C. Berg. E. Coli in Motion. Springer Verlag, New York, 2004.

[3] B.M Haines, I.S. Aranson, L. Berlyand and D.A. Karpeev. Effective viscosity of dilute bacterial suspensions: a two-dimensional model. Physical Biology, 5 (2008), No. 4.

[4] P. G. Ciarlet. Introduction à l'analyse numérique matricielle et à l'optimisation. Masson, Paris, 1990.

[5] L.H. Cisneros, R. Cortez, C. Dombrowski, R.E. Goldstein, J.O. Kessler. Fluid dynamics of self-propelled microorganisms. from individual to concentrated populations. Exp Fluids, 43 (2007), 737-753.

[6] Darnton NC, Turner L, Rojevsky S, Berg HC. Dynamics of bacterial swarming. Biophys J. 98 (2010), No. 10, 2082-90.

[7] A. Decoene, A. Lorz, S. Martin, B. Maury, M. Tang. Simulation of self-propelled chemotactic bacteria in a Stokes flow. ESAIM: Proc, 30 (2010), 104-123 .

[8] C. Dombrowski, L. Cisneros, S. Chatkaew, R. E. Goldstein, J. O. Kessler. Self-concentration and large-scale coherence in bacteria dynamics. Phys. Rev. Lett., 93 (2004), No. 9.

[9] D. Gérard-Varet, M. Hillairet. Regularity Issues in the Problem of Fluid Structure Interaction. to appear in Arch. Rational Mech. Anal.

[10] R. Glowinski, T. W. Pan, T. I. Hesla, D. D. Joseph \& J. Périaux. A fictitious domain approach to the direct numerical simulation of incompressible viscous flow past moving rigid bodies: application to particulate flow. J. Comp. Phys., 169 (2001), 363-427.

[11] R. Glowinski. Finite element methods for incompressible viscous flow. In: Handbook of $\mathrm{Nu}-$ merical Analysis, Vol. IX, P. G. Ciarlet and J.-L. Lions eds., Ed. North-Holland, Amsterdam, 2003. 
[12] V. Gyrya, K. Lipnikov, I. Aranson, L. Berlyand. Effective shear viscosity and dynamics of suspensions of micro-swimmers from small to moderate suspensions. Journal of Mathematical Biology (accepted, 2011).

[13] Hernandez-Ortiz J.P., C. Stoltz and M.D. Graham. Transport and col lective dynamics in suspensions of confined swimming particles. Phys. Rev. Lett., 95 (2005), pp. 204501.

[14] J. Happel, H. Brenner. Low Reynolds Number Hydrodynamics. Dordrecht, Kluwer, 1991.

[15] M. Hillairet. Lack of collision between solid bodies in a 2D constant-density incompressible flow. Communications in Partial Differential Equations 32 (2007), 1345-1371.

[16] J. Janela, A. Lefebvre, B. Maury. A penalty method for the simulation of fluid-rigid body interaction. ESAIM: Proc., 1 (2007), 115-123.

[17] D. Kaiser. Bacterial swarming, a re-examination of cell movement patterns. Curr Biol, 17 (2007),R561-R570.

[18] S. Kim, S.J. Karrila. Microhydrodynamics: Principles and Selected Applications. Dover, New York, 2005.

[19] E. Lauga and T.R. Powers. The hydrodynamics of swimming microorganisms. Rep. Prog. Phys., 72 (2009).

[20] A. Lefebvre. Fluid-particle simulations with Freefem++. ESAIM: Proc., 18 (2007), 120-132.

[21] A. Lefebvre, B. Maury. Apparent viscosity of a mixture of a Newtonian fluid and interacting particles. Fluid-solid interactions: modeling, simulation, bio-mechanical applications. Comptes Rendus Mcanique, 333 (2005), No. 12.

[22] B. Maury. A time-stepping scheme for inelastic collisions. Numerische Mathematik, 102 (2006), No. 4, 649-679.

[23] B. Maury. Numerical Analysis of a Finite Element / Volume Penalty Method. SIAM J. Numer. Anal. 47 (2009), No. 2, 1126-1148.

[24] J.T. Locsei, T.J. Pedley. Run and Tumble in Chemotaxis in a Shear Flow; The Effect of Temporal Comparisons, Persistence, Rotational Diffusion, and Cell Shape. Bulletin of Mathematical Biology, 71 (2009), 1089-1116.

[25] J.O. Kessler, T.J. Pedley. Hydrodynamic phenomena in suspensions of swimming microorganisms. Annu. Rev. Fluid Mech. 24 (1992), 313-58.

[26] F. Peruani, L. G. Morelli. Self-propelled particles with fluctuating speed and direction of motion in two dimensions. PRL 99 (2007), 010602, 2007.

[27] S. Rafai, L. Jibuti, P. Peyla. Effective viscosity of microswimmer suspensions. Phys. Rev. Lett., 104 (2010), 098102. 
[28] D. Saintillan, M. J. Shelley. Orientational order and instabilities in suspensions of selflocomoting rods. Phys. Rev. Lett., 99 (2007), 058102.

[29] J. E. Segall, S.M. Block, H.C. Berg. Temporal comparisons in bacterial chemotaxis. Proc. Natl . Acad. Sci. USA, 83 (1986), 8987-8991.

[30] A. Sokolov, I. S. Aranson. Reduction of viscosity in suspension of swimming bacteria. Phys. Rev. Lett. 103 (2009), 148101.

[31] A. Sokolov, R. E. Goldstein, F. I. Feldchtein, and I. S. Aranson. Enhanced mixing and spatial instability in concentrated bacterial suspensions. Phys. Rev. E 80 (2009), 031903.

[32] R. Temam, A. Miranville. Mathematical modeling in continuum mechanics. Cambridge University press, 2001.

[33] L. Turner, W.S. Ryu, H.C. Berg. Real-time imaging of fluorescent flagellar filaments. J. Bacteriol., 182 (2000), No. 10, 2793-2801.

[34] I. Tuval, L. Cisneros, C. Dombrowski, C. W. Wolgemuth, J.O. Kessler, R .E. Goldstein. Bacterial swimming and oxygen transport near contact lines. Proc. Natl. Acad. Sci. USA, 102 (2005), 2277-2282.

[35] S. Vincent, J. P. Caltagirone, P. Lubin \& T. N. Randrianarivelo. An adaptative augmented Lagrangian method for three-dimensional multimaterial flows. Computers and Fluids, 33 (2004), 1273-1289.

[36] X.-L. Wu, A. Libchaber. Particle diffusion in a quasi-two-dimensional bacterial bath. Physical Review Letters, 84 (2000), 3017-3020.

[37] Y. Wu, D. Kaiser, Y. Jiang, M. S. Alber. Periodic reversal of direction allows Myxobacteria to swarm. Proc. Natl. Acad. Sci. USA, 106 (2009), No. 4, 1222-1227. 